\title{
Symptômes reliés au diagnostic du trouble de personnalité limite à l'adolescence : une recension systématique de la littérature
}

\author{
par Élodie Le Bœuf \\ Département de psychologie \\ Faculté des arts et des sciences \\ Mémoire présenté \\ en vue de l'obtention du grade de maître \\ en Psychologie
}

Avril 2019

(C) Élodie Le Bœuf, 2019 


\section{Résumé}

Le diagnostic du trouble de personnalité limite (TPL) à l'adolescence est sujet de controverse chez certains chercheurs et cliniciens. Pour plusieurs, les symptômes présentés chez les jeunes ayant un TPL ressemblent au fonctionnement normal à l'adolescence. Le diagnostic est alors retardé à l'âge adulte, décalant la mise en place d'un traitement. L'objectif de cette recension systématique de la littérature est d'examiner les symptômes permettant la différenciation d'une population adolescente avec un TPL de celles ayant un autre diagnostic ou non psychiatrisée. En tout, 17 études sur les 4789 titres initialement identifiés rencontrent les critères d'inclusion et sont analysées. Cette recension systématique considère plusieurs symptômes diversifiés : troubles internalisés et externalisés, suicide, erreurs de mentalisation, schémas mésadaptés et diagnostics comorbides. Plusieurs symptômes distinguant de façon statistiquement significative les adolescents avec un TPL ont pu être observés en les comparant à trois groupes contrôles : les adolescents non psychiatrisés, ceux avec un autre trouble de personnalité et ceux ayant un autre diagnostic. Finalement, un patron de symptômes a pu également être observé entre les adolescents suicidaires ayant un TPL et ceux sans ce diagnostic.

Mots-clés: trouble de personnalité limite, adolescence, symptôme, comorbidité 


\begin{abstract}
The diagnosis of borderline personality disorder (BPD) in adolescence is controversial among some researchers and clinicians. For many, the symptoms presented in young people with BPD resemble normal functioning in adolescence. The diagnosis is then delayed in adulthood, postponing the treatment. The aim of this systematic review of the literature is to investigate the symptoms that can differentiate an adolescent population with BPD from one with another diagnosis and/or without any psychiatric issues. In all, 17 studies out of the 4,789 titles initially identified met the inclusion criteria and were analyzed. This systematic review considers diverse symptoms: internalized and externalized disorders, suicide, mentalization errors, maladaptive schemas and comorbid diagnoses. Several symptoms significantly differentiated adolescents with BPD from three control groups teenagers: non-psychiatric, with another personality disorder and with another diagnosis. Finally, a pattern of symptoms could also be observed between suicidal adolescents with BPD and suicidal youth without it.
\end{abstract}

Keywords: borderline personality disorder, adolescence, symptoms, comorbidity 


\section{Table des matières}

$\begin{array}{ll}\text { Introduction } & 7\end{array}$

$\begin{array}{ll}\text { Méthode } & 18\end{array}$

$\begin{array}{lc}\text { Résultats } & 18\end{array}$

$\begin{array}{ll}\text { Discussion } & 49\end{array}$

$\begin{array}{ll}\text { Conclusion } & 53\end{array}$

Références $\quad 54$ 


\section{Liste des tableaux}

Tableau I 12

Description du TPL selon la Classification internationale des maladies

Tableau II

Critères diagnostiques du TPL selon le Manuel diagnostique et statistique des troubles mentaux

Tableau III

Caractéristiques des études

Méthodologie des études 


\section{Liste des figures}

Figure 1

Démarche de sélection des études 


\section{Liste des abréviations}

DM: Dépression majeure

DSM: Diagnostic and statistical manual of mental disorders

TPL: Trouble de personnalité limite 


\section{Introduction}

Le trouble de personnalité limite (TPL) fait partie de la littérature scientifique depuis l'avènement du mouvement psychanalytique. Officiellement reconnu en 1980 dans la troisième version du Diagnostic and Statistical Manual of Mental Disorders (DSM), ce diagnostic se retrouve maintenant la cible de controverses. Le diagnostic du trouble, initialement réservé aux adultes, est maintenant attribué aux adolescents si les symptômes durent depuis au moins un an (American Psychiatric Association (APA), 1994; 2013). Cependant, plusieurs cliniciens hésitent encore à le reconnaître malgré les nombreuses études confirmant l'importance d'un diagnostic précoce.

Le but de cette recension systématique est de faire le point sur l'état des lieux concernant les patrons symptomatiques chez les adolescents ayant un diagnostic de TPL. Nous brosserons ainsi un portrait historique du TPL et discuterons de l'évolution des critères diagnostiques, de la prévalence du TPL chez les adolescents, des diverses raisons poussant les cliniciens à hésiter quant au diagnostic du TPL à l'adolescence et des conséquences à ne pas diagnostiquer rapidement. Également, nous désirons observer quels symptômes permettent la différenciation des adolescents avec ce diagnostic de ceux ayant d'autres troubles psychologiques. À l'aide de cette recension, nous souhaitons faciliter autant la reconnaissance du TPL que la différenciation entre celui-ci et les autres troubles qui s'y apparentent, particulièrement lors de l'adolescence.

\section{Historique du trouble de personnalité limite}

C'est en 1938 qu'Adolph Stern mentionne pour la première fois un groupe de patients dont les symptômes se situent à la limite entre la psychose et la névrose, d'où son appellation condition limite (borderline). Les traitements traditionnels psychanalytiques s'avèrent inutiles pour guérir 
ces patients. Stern décrit ces symptômes cliniques en dix traits: narcissisme, hémorragie psychique, hypersensibilité excessive, rigidité psychique, réactions thérapeutiques négatives, sentiment d'infériorité, masochisme, insécurité/anxiété somatique, mécanismes de projection et difficultés dans l'épreuve de réalité. Malgré une description large et vétuste du trouble limite, appelé comme tel à l'époque, plusieurs éléments mentionnés par Stern sont encore utilisés aujourd'hui. Tout d'abord, Stern (1938) observe la présence de difficultés durant l'enfance de ses patients, dont un certain nombre aurait été témoins de conflits, de séparation, de divorce et d'abandon par leurs parents. La cruauté, l'abus et la négligence répétés se retrouvent également chez ces patients. Stern constate aussi que ces personnes ont une personnalité rigide en réponse à une expérience anxiogène. Au cours de la thérapie, les interprétations amenées par le thérapeute sont mal reçues et plongent le patient dans la dépression, le découragement ou peuvent provoquer une colère soudaine et un sentiment de rejet. Stern mentionne toutefois que ces personnes peuvent avoir l'air étrangement sereines malgré la présence de situations difficiles. De plus, leur interprétation des événements est très clivée, variant entre l'autodénigrement et une exagération éphémère de leur estime de soi. Finalement, Stern (1938) fait le lien entre le trouble limite et la psychose en mentionnant la tendance de ces patients à l'utilisation du mécanisme de défense de la projection. Selon lui, afin de maitriser plus facilement les causes de leur anxiété, ces personnes projettent celles-ci sur leur environnement.

En 1945, Stern publie ses observations concernant l'origine de cette condition limite particulière. Ainsi, la condition borderline serait provoquée par un environnement continuellement traumatisant et invalidant, situation à laquelle l'enfant serait incapable de faire face, amenant par la suite, à l'âge adulte, le développement d'une certaine appréhension, ou même un réflexe, face aux dangers anticipés dans leur environnement et leurs relations interpersonnelles. Selon Stern, un 
enfant élevé par un ou des parents invalidants et démontrant peu d'amour envers lui développe une peur de la punition ou du rejet par son entourage, ainsi qu'un besoin exagéré d'être entendu et respecté. À l'âge adulte, ce besoin peut se transformer en une fixation malsaine, la personne cherchant ainsi sans arrêt l'affection et le support dans ses relations interpersonnelles. Enfin, Stern mentionne les nombreuses difficultés rencontrées en thérapie avec cette clientèle, ainsi que la nécessité de modifier la thérapie psychanalytique traditionnelle pour aider ces patients particuliers.

Pour sa part, Helene Deutsch (1942) entreprend la description d'un type de personnalité qu'elle nomme " comme si » ("as-if"). Elle observe alors que les gens ayant ce type de personnalité sont dépendants, c'est-à-dire qu'ils modifient leurs comportements, leurs attitudes, leurs croyances et leurs valeurs selon la rétroaction de l'environnement et de leur entourage. Leurs idéaux et leurs convictions ne reflètent alors que ceux des autres, pouvant provoquer un sentiment profond de vide et de toute absence de sincérité. Selon Deutsch, ces personnes s'accrochent rapidement au moindre objet d'identification. Les relations interpersonnelles sont alors très intenses à leurs débuts, amenant une certaine gratification au partenaire ou à l'objet d'identification. La personne 'as-if" adopterait, tel un caméléon, les mêmes caractéristiques que son partenaire. Avec le temps, un certain manque de sincérité et de chaleur dans la relation provoque un vide pouvant déboucher sur la fin de la relation. Cet abandon et le sentiment de rejet vécu alors par la personne pourraient occasionner soit une réaction intense afin de demeurer accroché au partenaire, soit la recherche immédiate d'un nouvel objet d'identification. Deutsch (1942) mentionne également que les personnes "as-if"' ne semblent pas conscientes de leur réactivité affective ou de leurs liens interpersonnels anormaux, que leur entourage ne manque pourtant pas de remarquer. Au contraire, ces perturbations émotionnelles et relationnelles sont perçues comme faisant simplement partie de leur personnalité ou sont simplement projetées sur 
l'environnement. Finalement, étant donné qu'il y a utilisation de mécanismes de défense primitifs tel que le clivage, et vu le bon ajustement de ces personnes à l'épreuve de la réalité, Deutsch affirme que ce type de personnalité ne correspond ni à la catégorie des troubles névrotiques, ni à celle des troubles psychotiques.

Au cours des années 50, plusieurs chercheurs tentent de démystifier le concept d'état limite. La description des symptômes proposée par Robert Knight (1952) et Arlene Robbins Wolberg (1952) ressemblent plutôt au trouble de la personnalité schizotypique. De plus, Melitta Schmideberg (1959) apporte l'idée d'une population particulière qui semble stable dans son instabilité, similaire aux schizophrènes en rémission (New et Triebwasser, 2017). Ce n'est qu'en 1966 que Otto Kernberg entreprend la description et la définition de cette pathologie limite. Selon lui, une organisation de la personnalité limite est un trouble possédant une certaine stabilité temporelle et dont les symptômes se rapprochent plutôt de la névrose que la psychose. Kernberg observe que les patients ayant ce type d'organisation de la personnalité ont en commun une certaine impulsivité sélective et contradictoire. Plus spécifiquement, cette absence de contrôle de l'impulsivité camoufle plutôt une alternance des comportements contradictoires, de clivage. Kernberg donne en exemple l'un de ses patients qui, semaine après semaine, alterne entre une attitude de dévalorisation et d'idéalisation par rapport à leur relation. Les personnes possédant une organisation de la personnalité limite pourraient être conscientes de ces comportements, mais nient leurs impacts et ne semblent pas s'en inquiéter. Ils semblent posséder une perception dichotomique de leur environnement. La réalisation de l'importance de ces contradictions peut provoquer une forte anxiété. Ainsi, l'utilisation de ce mécanisme de défense (clivage) ne serait qu'une protection contre l'angoisse et serait occasionnée par un conflit interne d'émotions ne pouvant cohabiter. 
En 1968, Roy Grinker, un des premiers chercheurs à tenter de définir empiriquement la personnalité limite, identifie quatre facteurs: colère, dépression, absence d'une identité stable et difficulté dans les relations interpersonnelles. Finalement, à la suite de l'ajout officiel du trouble de personnalité limite dans la troisième version du DSM, John Gunderson (1981) publie le premier instrument diagnostique spécifique au TPL, le Diagnostic Interview for Borderline Personality Disorder (DIB), encore utilisé aujourd'hui. Depuis, la communauté scientifique s'est grandement penchée sur l'étiologie, la symptomatologie et l'aspect social et biologique de ce trouble (New et Triebwasser, 2017).

\section{Évolution des critères diagnostiques}

Tel que signalé précédemment, le TPL contemporain fut officiellement ajouté dans la troisième édition du DSM. Il est tout de même possible de retrouver des diagnostics précurseurs au TPL dans le DSM-I (1952) et le DSM-II (1968). Effectivement, plusieurs symptômes du TPL inclus dans le DSM-IV ressemblent aux diagnostics de la personnalité émotionnelle instable ainsi que celui de la personnalité cyclothymique notés dans le DSM-I. Dans le DSM-II, le diagnostic de la personnalité émotionnelle instable est retiré, le trouble de personnalité cyclothymique demeure et le trouble de personnalité explosive est ajouté (New et Triebwasser, 2017). C'est seulement à partir du DSM-III (1980) que les définitions d'un trait de personnalité et un trouble de personnalité général sont mentionnées. Un trait de personnalité représente un schéma cognitif, perceptif et relationnel englobant soi-même et l'environnement externe présent dans plusieurs contextes personnels et sociaux. Un trouble de personnalité (TP) est présent lorsque ces traits de personnalité deviennent mésadaptés, rigides et causent des problèmes relationnels, sociaux et fonctionnels ainsi qu'une détresse significative (APA, 1980, 1994, 2013). 
Six critères plus formels définissant le trouble de la personnalité général sont ajoutés au DSM-IV. Tout d'abord, il doit y avoir la présence d'un patron perceptif et comportemental persistant qui dévie des attentes culturelles du patient dans au moins deux domaines suivants: la cognition, le contrôle des impulsions, les affects et le fonctionnement interpersonnel (critère A).

De plus, ce patron est inflexible et envahissant (critère B), provoque une souffrance cliniquement significative et une altération du fonctionnement normal (critère C). Le patron est stable, durable et débute à la fin de l'adolescence ou au début de l'âge adulte (critère D). Finalement, ce patron dysfonctionnel n'est pas mieux expliqué par les effets d'un autre trouble mental (critère E), ni des effets physiologiques d'une substance (drogue, médicament, substance toxique) ou d'un malaise médical (critère F) (APA, 1994, 2013). Les critères selon la Classification internationale des maladies (CIM-10, 1993) se retrouvent au tableau I. L'évolution des critères diagnostiques à partir du DSM-III jusqu'au DSM-5 se retrouve au tableau II.

\section{Tableau I - Description du TPL selon la Classification internationale des maladies}

\section{CIM-10 (1993)}

Trouble de la personnalité émotionnellement labile, type borderline

1. Instabilité émotionnelle, colère intense menant à la violence

2. Présence de comportements impulsifs sans réflexion aux conséquences possibles

3. Difficulté à planifier sur le long-terme

4. Perturbation de l'image de soi, des buts et des préférences personnelles

5. Sentiment chronique de vide

6. Relations interpersonnelles instables et intenses pouvant être associées à des efforts pour éviter l'abandon, des menaces suicidaires ou de l'automutilation 
Tableau II - Critères diagnostiques du TPL selon le Manuel diagnostique et statistique des troubles mentaux

$$
\text { DSM-III (1980) DSM-IV (1994) et DSM-5 (2013) }
$$

\begin{tabular}{|c|c|}
\hline Au moins cinq des critères suivants; & Au moins cinq des critères suivants; \\
\hline $\begin{array}{l}\text { 1. Impulsivité dans au moins deux sphères } \\
\text { pouvant être autodestructrices }\end{array}$ & $\begin{array}{l}\text { 1. Efforts pour éviter un abandon réel ou } \\
\text { imaginaire (ne pas inclure le critère } 5 \text { ) }\end{array}$ \\
\hline $\begin{array}{l}\text { 2. Relations interpersonnelles intense et } \\
\text { instable (idéalisation, dévalorisation, } \\
\text { manipulation, changement d'attitude) }\end{array}$ & $\begin{array}{l}\text { 2. Relations interpersonnelles instables et } \\
\text { intenses avec une alternance de positions } \\
\text { extrêmes entre l'idéalisation excessive et la }\end{array}$ \\
\hline inappropriée et difficulté à & 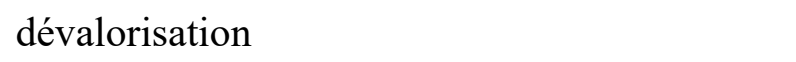 \\
\hline & Ginctabilitó mo \\
\hline $\begin{array}{l}\text { 4. Identité perturbée (image de soi, identité de } \\
\text { genre, buts à long-terme, carrière, valeurs) }\end{array}$ & $\begin{array}{l}\text { et persistante de l'image ou de la notion de } \\
\text { soi) }\end{array}$ \\
\hline $\begin{array}{l}\text { 5. Instabilité affective : soudains changements } \\
\text { qui durent de quelques heures à quelques } \\
\text { jours avec un retour à la normale }\end{array}$ & $\begin{array}{l}\text { 4. Impulsivité dans au moins deux domaines } \\
\text { potentiellement autodestructeurs (ne pas } \\
\text { inclure le critère 5) }\end{array}$ \\
\hline $\begin{array}{l}\text { 6. Intolérance à la solitude avec efforts pour } \\
\text { éviter d'être seul }\end{array}$ & $\begin{array}{l}\text { 5. Répétition de comportements, gestes ou } \\
\text { menaces suicidaires ou automutilations }\end{array}$ \\
\hline $\begin{array}{l}\text { 7. Comportements autodestructeurs tels que } \\
\text { des gestes et des menaces suicidaires, }\end{array}$ & $\begin{array}{l}\text { 6. Instabilité affective due à une réactivité } \\
\text { marquée de l'humeur (épisode dysphorique, }\end{array}$ \\
\hline & \\
\hline & \\
\hline roniques de & $\begin{array}{l}\text { 8. Colère intense et inappropriée et une } \\
\text { difficulté à la contrôler }\end{array}$ \\
\hline $\begin{array}{l}\text { B. Si le patient n'a pas atteint } 18 \text { ans, il ne } \\
\text { doit pas remplir les critères pour le trouble } \\
\text { identitaire }\end{array}$ & $\begin{array}{l}\text { 9. Survenue transitoire d'idéation persécutoire } \\
\text { ou des symptômes dissociatifs sévères en } \\
\text { période de stress }\end{array}$ \\
\hline
\end{tabular}

Le TPL est un diagnostic trois fois plus présent chez les femmes que les hommes. Les symptômes débuteraient au début de la puberté, augmenteraient en nombre et en intensité entre 14 et 17 ans et finiraient par diminuer au cours de l'âge adulte. Selon quelques études, $0,9 \%$ des adolescents présentent les critères diagnostiques. Le pourcentage augmenterait ainsi à 1,4\% à 16 ans et jusqu'à $3,2 \%$ à l'âge de 22 ans. Environ 3\% des adolescents dans la communauté présenteraient les critères du TPL. Jusqu'à 50\% des adolescents hospitalisés et environ $11 \%$ des adolescents psychiatriques non hospitalisés sont diagnostiqués avec un TPL. Le TPL est ainsi un 
trouble relativement présent dans la population adolescente et extrêmement prévalent chez les jeunes hospitalisés (Kaess, Brunner et Chanen, 2014; Courtney-Seidler, Klein et Miller, 2013).

\section{Réticences au diagnostic du TPL à l'adolescence}

Les cliniciens et les chercheurs hésitent à diagnostiquer un TPL chez les adolescents pour au moins quatre raisons: la validité et la stabilité du diagnostic, la stigmatisation du terme « personnalité limite » et la théorie du développement de la personnalité (Kaess et al., 2014). Quelques critères du TPL ressemblant à certaines caractéristiques du développement normal à l'adolescence, plusieurs cliniciens craignent d'apposer un diagnostic aussi lourd à quelqu'un traversant simplement un épisode de vie difficile à l'adolescence. Certains mentionnent également l'influence qu'un tel diagnostic puisse avoir sur le développement de l'identité et du concept de soi d'un adolescent, ainsi que sur la perception de l'entourage (Courtney-Seidler et al., 2013).

En ce qui concerne la validité du diagnostic du TPL, celle-ci peut être questionnée: mesuret-on réellement un TPL ou un autre trouble? Effectivement, plusieurs symptômes du TPL ressemblent aux comportements et aux affects normalement présents à l'adolescence. La sévérité et la persistance des symptômes, ainsi que la perturbation au quotidien provoquée par ceux-ci permettraient de différencier un adolescent sain d'un adolescent ayant un TPL (APA, 1994; 2013). Dans leur revue de la littérature spécifique à la validité et à la fiabilité du diagnostic du TPL, Miller, Muehlenkamp et Jacobson (2008) discutent, premièrement, de la validité du TPL en tant que construit. Selon eux, le trouble de personnalité limite serait particulièrement lié à certaines perturbations psychologiques et comportementales susceptibles d'être observées depuis le début de l'adolescence. De plus, la validité du diagnostic à l'adolescence serait équivalente à celle du diagnostic chez l'adulte. Selon les études relevées dans leur revue de la littérature, les chercheurs n'observent aucune différence relative à l'efficacité des critères du TPL entre les adolescents et 
les adultes. Ainsi, les critères diagnostiques utilisés chez l'adulte seraient aussi valides chez l'adolescent. Par ailleurs, la validité prédictive du diagnostic observée dans les études était de modeste à modérée. La majorité des adolescents ne satisfaisant plus les critères diagnostiques au follow-up conservaient cependant un certain niveau de dysfonction sous-clinique. Ce phénomène était également observé chez les adultes. Finalement, la sensibilité et la prédictibilité des critères diagnostiques étaient comparables chez les adultes et les adolescents. Les résultats semblent ainsi pointer vers une validité modérée du diagnostic de TPL à l'adolescence tout en demeurant similaire à celle retrouvée chez les adultes (Courtney-Seidler et al., 2013).

Pour leur part, Miller et collaborateurs (2008) discutent de la stabilité du TPL. Selon les études rapportées, un grand nombre de patients identifiés avec un TPL ne conserve pas leur diagnostic. Ils maintiennent cependant un nombre sous-clinique de symptômes. Le nombre de jeunes gardant leur diagnostic est néanmoins statistiquement significatif. La persistance du diagnostic est relativement basse, mais est similaire à celle observée chez les adultes. Effectivement, la stabilité du diagnostic à l'adolescence (14\%-40\%) chevaucherait celle des adultes (25\%-67\%). En fait, ce serait l'intensité des symptômes qui varierait et non le concept en soi du TPL, puisque celui-ci peut être présent à un niveau sous clinique autant à l'adolescence qu'à l'âge adulte. (Winsper et al., 2015)

La stigmatisation est également un élément à prendre en compte avant d'apposer le diagnostic d'un TPL chez un jeune adolescent. Effectivement, selon l'étude de Catthoor, Feenstar, Hutsebaut, Schrijvers et Sabbe (2015), les adolescents cherchant de l'aide thérapeutique pour un trouble de personnalité vivent plus de stigmatisation que ceux ayant n'importe quel autre diagnostic de l'axe I. Plus spécifiquement, le diagnostic d'un TPL est le plus grand prédicteur de stigmatisation en comparaison aux autres troubles de personnalité. Cependant, Chanen (2015) 
apporte l'idée que le refus d'utiliser le TPL comme diagnostic potentiel à l'adolescence à cause de la stigmatisation ne fait plutôt que renforcer les stéréotypes reliés à ce diagnostic.

Finalement, la théorie du développement de la personnalité chez les adolescents est également mentionnée par certains cliniciens comme une raison d'éviter un diagnostic de TPL à l'adolescence. Tel que discuté par Costa et McCrae (1994), la personnalité ne parviendrait à maturité qu'à 30 ans. Selon les deux auteurs, la structure du tempérament chez l'enfant serait un précurseur des cinq grands facteurs de la personnalité de l'adulte (Big Five: ouverture, conscience, extraversion, agréabilité et névrotisme) et il faudrait ainsi parler de la continuité du développement de la personnalité plutôt que de la stabilité de celle-ci. Les variations de la personnalité avec l'âge s'observeraient par l'alternance de la position des individus sur les éléments de base de la personnalité plutôt que sur la structure même (Costa et McCrae, 1994). Ainsi, malgré certains changements concernant la personnalité à l'adolescence, les individus conserveraient une structure de base stable à travers le temps, pouvant rendre le diagnostic du trouble de personnalité limite relativement possible avant l'âge adulte.

\section{Conséquences d'un diagnostic tardif}

Le TPL est associé à plusieurs dysfonctionnements comportementaux et psychologiques pouvant mener à des conséquences majeures s'ils ne sont pas traités. Les comportements suicidaires, par exemple, sont particulièrement fréquents chez cette population. Entre $8 \%$ et $10 \%$ des patients diagnostiqués avec un TPL se suicident, environ 75\% des patients font des tentatives de suicide non létales et jusqu'à $70 \%$ font des tentatives suicidaires pouvant être mortelles (Laporte et Desrosiers, 2016; Oldham, 2006). Plus spécifiquement dans la population adolescente, les comportements suicidaires sont présents chez $80 \%$ des patients et $30 \%$ des adolescents morts par suicide présentent les critères du TPL. Les comportements suicidaires commencent également plus 
tôt et le nombre de tentatives de suicide est plus nombreux chez les jeunes ayant un TPL que la dépression. (Laporte et Desrosiers, 2016) L'impulsivité étant également fréquente chez cette population, plusieurs jeunes s'engagent dans des comportements risqués, tels l'abus de substance, la sexualité non protégée et l'automutilation (Oldham, 2006). Certains vont jusqu'à développer certains troubles alimentaires ou font des menaces suicidaires (Laporte et Desrosiers, 2016). À long terme, cette population démontre plus de difficultés scolaires, utilise plus l'aide sociale et manque davantage de jours de travail. Un diagnostic de TPL est également associé à certains troubles de santé physiques tels que les troubles cardiovasculaires, l'arthrite et les problèmes gastro-intestinaux (Oldham, 2006). Finalement, ne pas recevoir de traitement spécifique rapide ou entreprendre un traitement inapproprié peut exacerber les problèmes comportements et psychologiques mentionnés plus tôt (Miller, Muehlenkamp et Jacobson, 2008). Une intervention rapide grâce au diagnostic à l'adolescence peut permettre d'interrompre le développement de comportements dysfonctionnels et empêcher la cristallisation de ceux-ci, rendant de futures interventions plus difficiles.

Le diagnostic du TPL semble difficile à utiliser dans une population adolescente. Il est ainsi nécessaire de déterminer les symptômes permettant de différencier les jeunes ayant un TPL des autres adolescents. Notre recension systématique a pour but d'identifier un patron symptomatique chez les adolescents ayant un TPL en comparant cette population aux adolescents ayant un autre diagnostic et ceux non psychiatrisés. 


\section{Méthode}

Pour réaliser cette recension systématique, trois bases de données ont été utilisées: PubMed, PsycInfo et Web of Science. Les termes "borderline personality disorder" AND adolescen* OR child* OR youth OR young OR juvenil* OR teen* AND symptom* ont été utilisés dans les bases de données. La recherche systématique, complétée à la fin de mai 2018, s'est attardée aux études réalisées depuis 1980, lorsque le trouble de personnalité limite fut officiellement ajouté au DSM-III. Seules les études en anglais et en français ont été retenues.

\section{Sélection des études}

Les critères d'inclusion utilisés pour la recherche systématique sont les suivants :

1. Études utilisant un échantillon adolescent, dont l'âge se situe entre 12 et 18 ans.

2. Études qui présentent des symptômes et/ou troubles comorbides internalisés et/ou externalisés.

3. Études qui comparent un échantillon ayant un diagnostic de TPL à un échantillon psychiatrique sans TPL et/ou à un échantillon de la population non psychiatrique.

4. Études quantitatives comparatives.

\section{Résultats}

Un total de 4789 études a pu être identifié à l'aide des trois bases de données, dont 2248 études ont été répertoriées dans PsycNet, 1139 études dans PubMed et 1402 études dans Web of Science. Ces études ont ensuite été placées dans le programme de gestion de références bibliographiques EndNote, ce qui a permis de retirer les doublons: 1952 titres ont alors été exclus. À la suite de la lecture de tous les titres et résumés, 2667 études n'ont pas été retenues puisqu'elles 
ne respectaient pas les critères d'inclusion présentés plus tôt. Ainsi, 170 études ont été analysées dans leur entièreté afin de s'assurer qu'elles possèdent tous les critères d'inclusion. À la suite de l'analyse complète des textes, 153 études ont été exclues de la revue: 37 études parce qu'elles ne discutent pas spécifiquement du TPL, 24 puisqu'elles n'ont pas utilisé un échantillon adolescent, 21 études ne possèdent pas de groupes comparatifs, 18 études n'observent pas des symptômes ou des troubles internalisés et/ou externalisés, 24 études suivent un devis de recherche longitudinal, 17 études non quantitatives, 10 études sont des revues de la littérature non pertinentes et 2 études ont été écrites dans une autre langue que le français ou l'anglais. La figure 1 fournit le processus de sélection des études scientifiques.

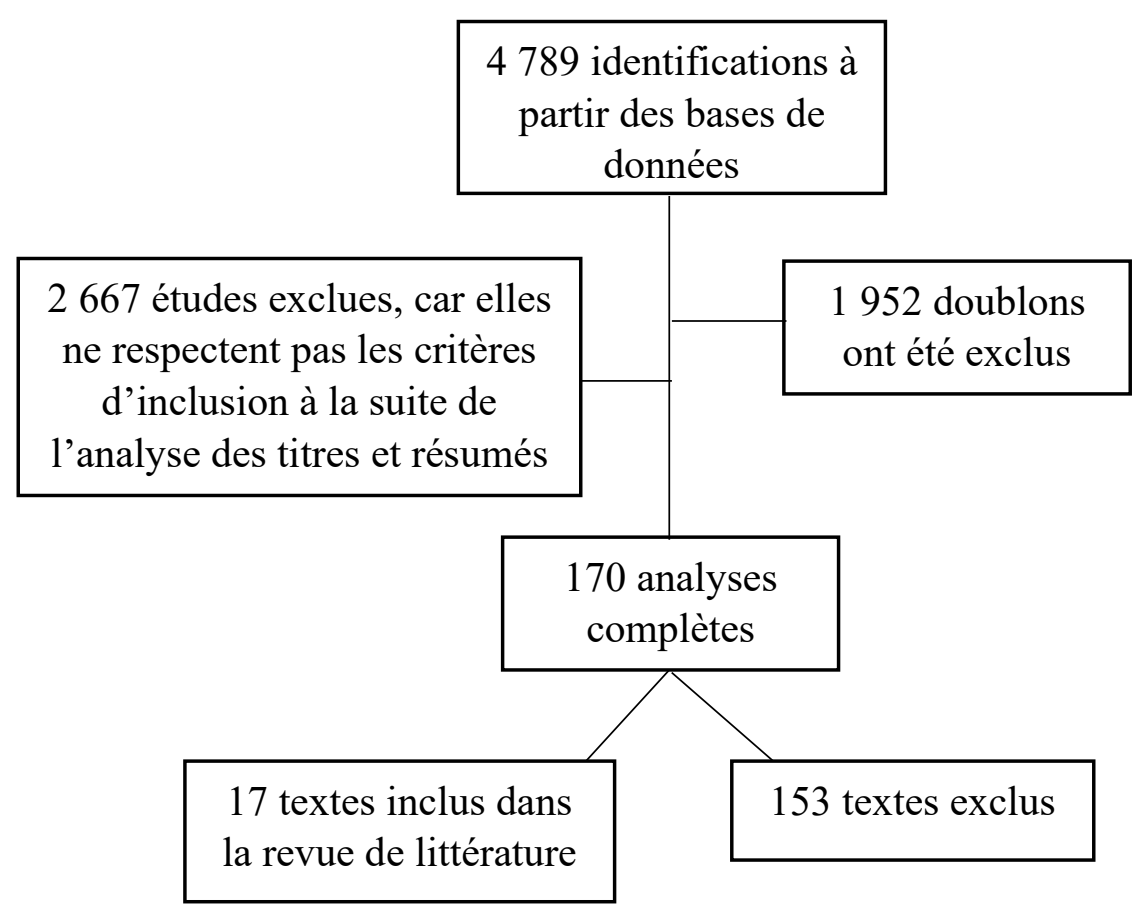

Figure 1. Démarche de sélection des études 
Les 17 études rapportées dans notre recension systématique sont présentées en ordre alphabétique. Les caractéristiques des études sont présentées dans le tableau III et les informations méthodologiques dans le tableau IV.

Atlas et Wolfson (1996) ont comparé un groupe de jeunes filles ayant un diagnostic de TPL à un groupe psychiatrique sans TPL au niveau de la dépression et de la dissociation. Les adolescentes dans le groupe TPL ont obtenu un résultat entre six et huit au Diagnostic Interview for Borderlines (DIB), alors que les autres ont été incluses dans le groupe contrôle. Afin de comparer les deux groupes sur les symptômes de la dépression et de la dissociation, les chercheurs ont utilisé le Beck Depression Inventory (BDI) et le Dissociative Experiences Scale Revised (DESR). Le groupe TPL montre des résultats significativement plus élevés au BDI $(M=21,6$ contre $M=7,8, t=4,2 p<0,001)$ et au DES-R $(M=32,8$ contre $M=8,5, t=4.5 p<0,001)$ que le groupe contrôle psychiatrique. Ainsi, les adolescentes ayant un trouble de personnalité limite avaient des résultats plus élevés au niveau de la dépression et de la dissociation.

Chabrol, Montovany, Callahan, Chouicha et Ducongé (2002) se sont penchés sur la validité du trouble de personnalité limite à l'adolescence. Pour ce faire, ils ont procédé à une analyse factorielle en utilisant le Revised Diagnostic Interview for Borderlines (DIB-R). Deux groupes ont donc été comparés: les adolescents répondant aux critères du TPL (groupe TPL) et ceux n'ayant pas dépassé le nombre de critères nécessaire (groupe sans trouble psychiatrique). Une première analyse (Test-t) a été faite afin de comparer les deux groupes sur les différentes sous-sections du DIB-R. Par la suite, trois facteurs ont été mis en évidence (Scree Test et rotation de facteurs de Varimax): affects et cognition, impulsivité et agressivité dans les relations interpersonnelles. Le premier facteur regroupait les symptômes de dépression $(E T=0,59, p<0,002)$, d'impuissance $(E T=0,00, p<0,002)$, d'anxiété, de solitude $(E T=0,45, p<0,002)$, de pensées étranges $(E T=0,78$, 
$p<0,002)$, d'expériences quasi psychotiques ( $E T=0,75, p<0,002)$, de comportements suicidaires ( $E T=0,70, p<0,002)$, d'intolérance à la solitude $(E T=0,78, p<0,002)$, de préoccupations d'abandon $(E T=0,83, p<0,002)$ et de dépendance. Une différence statistiquement significative a été trouvée à chaque sous-section, à l'exception de l'anxiété et la dépendance. Le deuxième facteur comportait les sous-sections de l'abus de substance, la déviance sexuelle, la contre-dépendance et des relations interpersonnelles difficiles. Seules les sous-sections de la contre-dépendance $(E T=0,78, p<0,002)$ et de la déviance sexuelle $(E T=1,02, p<0,002)$ étaient significativement différentes entre le groupe TPL et le groupe contrôle. Le troisième facteur réunissait les sous-sections de la colère, les symptômes hypomaniaques, l'automutilation, la manipulation et l'exigence. Seules les soussections de la manipulation ( $E T=0,92, p<0,002)$ et l'exigence $(E T=0,88, p<0,002)$ étaient statistiquement significatives entre les deux groupes. La sous-section des expériences de paranoïa non délirantes n'a été incluse dans aucun facteur. Cependant, il y avait une différence significative $(E T=0,61, p<0,002)$ entre le groupe TPL et le groupe contrôle. Finalement, les trois facteurs se sont révélés statistiquement significatifs entre le groupe TPL et le groupe contrôle (facteur 1: $t=-7,4, p<0,00001$; facteur 2: $t=-5,8, p<0,00001$; facteur 3: $t=-4,2, p<0,0001)$.

Pour leur part, Chanen, Jovev et Jackson (2007) ont voulu observer le fonctionnement adaptatif et la psychopathologie chez les adolescents ayant un TPL. Pour ce faire, ils ont comparé trois groupes de jeunes non hospitalisés: un groupe ayant un diagnostic de TPL, un groupe ayant un diagnostic d'un trouble de personnalité autre que le TPL et un groupe n'ayant aucun diagnostic d'un trouble de personnalité. Le Structured Clinical Interview for DSM-IV Axis I Disorders (SCIDI) ainsi que la section des troubles du comportement du Kiddie Schedule for Affective Disorders and Schizophrenia - Present and Lifetime Version (K-SADS-PL) ont été utilisés afin d'obtenir les diagnostiques de l'axe I. Les diagnostics concernant les troubles reliés à l'abus de drogues et 
d'alcool ont été faits à l'aide du Composite International Diagnostic Interview - Auto (CIDI-Auto).

Les troubles de la personnalité selon le DSM-IV ont été observés avec le Structured Clinical Interview for DSM-IV Axis II Disorders (SCID-II), les troubles internalisés et externalisés avec le Youth Self-Report (YSR) et le Young Adult Self-Report (YASR) et les éléments du fonctionnement psychosocial avec le Health of the Nation Outcomes Scales for Children and Adolescents (HoNOSCA) et le Social and Occupational Functioning Assessment Scale (SOFAS). Plusieurs différences statistiquement significatives ont été observées. Au niveau des diagnostics de l'axe I, le groupe TPL rapportait un nombre significativement plus élevé de troubles de l'humeur $\left(x^{2}=11,53, p<0,01\right)$, de troubles de comportements $\left(x^{2}=33,92, p<0,01\right)$, d'abus de substance $\left(x^{2}=15,93, p<0,01\right)$ et de troubles reliés à la consommation de nicotine $\left(x^{2}=32,81, p<0,01\right)$ que le groupe avec d'autres TP, ainsi que le groupe sans TP. Le nombre de troubles anxieux était significativement plus élevé chez le groupe TPL que chez le groupe sans TP seulement $\left(x^{2}=7,48\right.$, $p=0,02)$. Le groupe TPL a également rapporté un niveau plus élevé d'automutilation que le groupe sans trouble de personnalité $\left(x^{2}=24,15, p<0,01\right)$. Aucune différence significative n'a été rapportée au sujet des troubles de l'alimentation. Au niveau des résultats au SOFAS, le groupe TPL a présenté un moins bon degré de fonctionnement global que le groupe sans $\operatorname{TP}(F=13,48, p<0,01)$. Plus spécifiquement, concernant les raisons de l'incapacité des adolescents à poursuivre leurs activités quotidiennes, seul l'absentéisme scolaire fut rapporté significativement plus souvent chez le groupe TPL que chez le groupe avec d'autres TP $(F=3,14, p=0,05)$. Le groupe TPL rapportait également plus de problèmes internalisés $(F=20,71, p<0,01)$ et externalisés $(F=26,5, p<0,01)$ sur le YSR et le YASR que le groupe avec d'autres TP ainsi que le groupe sans TP. Finalement, une différence significative a été observée dans trois des quatre domaines du HoNOSCA. Le groupe avec TPL rapportait des résultats plus faibles que le groupe sans TP au niveau des relations 
interpersonnelles $(F=13,84, p<0,01)$, des soins personnels $(F=5,43, p=0,01)$ et des relations familiales $(F=6,88, p<0,01)$.

Goodman et collaborateurs $(2010,2013)$, ont entrepris deux études au sujet de la trajectoire développementale du TPL chez les personnes ayant un TPL. Pour se faire, ils ont créé un questionnaire que les parents pouvaient remplir sur internet concernant plusieurs aspects de la vie de leur enfant ayant un TPL en comparaison avec leurs frères ou sœurs n'ayant pas reçu ce diagnostic. Ce questionnaire, développé par les membres du Mount Sinai Mood and Personality Disorders Research Group, rapportait plusieurs éléments en partant de la période de la grossesse jusqu'au début de l'âge adulte. Afin de confirmer le diagnostic de TPL, le McLean Screening Instrument for Borderline Personality Disorder (MSI-BPD) fut ajouté au questionnaire. Leur première étude (Goodman et al., 2010) était centrée spécifiquement sur la trajectoire du TPL chez les filles en comparaison avec leur(s) sœurs(s) n'ayant pas ce diagnostic. Les plus grandes différences observées entre les deux groupes au MSI-BPD provenaient de cinq critères: sentiment de vide chronique, perturbation identitaire, effort constant pour éviter un abandon, sentiment d'irréel et automutilation. Les comportements impulsifs, la victimisation, l'automutilation, les préoccupations de l'image de soi, les caractéristiques psychotiques, les problèmes interpersonnels et les symptômes affectifs étaient les aspects comparés pour la période de l'adolescence. La prévalence de tous les éléments suivants était significativement plus élevée chez les filles ayant un TPL que leur(s) sœur(s) n'ayant pas de TPL: passage à l'acte $\left(x^{2}=68,726, p<0,000\right)$, promiscuité $\left(x^{2}=55,305, p<0,000\right)$, grossesse $\left(x^{2}=9,029, p<0,003\right)$, arrestation $\left(x^{2}=10,850, p<0,001\right)$, abus verbal $\left(x^{2}=64,524, p<0,000\right)$, impulsivité $\left(x^{2}=126,226, p<0,000\right)$, agression $\left(x^{2}=27,480, p<0,000\right)$, abus de substance $\left(x^{2}=37,151, p<0,000\right)$, idées suicidaires $\left(x^{2}=69,699, p<0,000\right)$, menaces suicidaires $\left(x^{2}=49,532, p<0,001\right)$, tentatives de suicide $\left(x^{2}=28,157, p<0,000\right)$, automutilation non 
suicidaire $\left(x^{2}=54,886, p<0,000\right)$, victime d'agression $\left(x^{2}=6,995, p<0,008\right)$, idées délirantes $\left(x^{2}=20,573, p<0,000\right)$ et anorexie $\left(x^{2}=11,384, p<0,001\right)$.

En 2013, Goodman, Patel, Oakes, Matho et Triebwasser ont poursuivi l'étude de 2010 en observant la trajectoire développementale du TPL chez les garçons. La même méthode de recherche fut utilisée. Les plus grandes différences entre les deux groupes au MSI-BPD variaient de celles observées chez les filles: sentiment de vide chronique, perturbation identitaire, effort constant pour éviter un abandon, impulsivité et difficultés relationnelles. Spécifiques à l'adolescence, les items du questionnaire abordaient les comportements impulsifs, la victimisation, l'automutilation, les perturbations de l'image de soi, les caractéristiques psychotiques, les problèmes interpersonnels et la labilité affective. Plusieurs éléments ont été démontrés significativement différents entre le groupe TPL et le groupe contrôle. Au niveau des comportements impulsifs, le nombre d'arrestations ( $O R=3,88, p<0,000)$, l'impulsivité ( $O R=24,99$, $p<0,000)$, le vol $(O R=12,26, p<0,000)$, l'abus d'alcool $(O R=7,28, p<0,000)$, l'abus de substance $(O R=9,5, p<0,000)$ sont quelques résultats observés. Les items de victimisation concernaient l'abus physique ( $O R=3,64, p<0,029)$, l'abus sexuel $(O R=4,14, p<0,013)$, être victime de violence $(O R=4,83, p<0,000)$ et être victime de viol $(O R=7,1, p<0,044)$. L'automutilation englobait les idées suicidaires $(O R=12,64, p<0,000)$, les menaces suicidaires $(O R=22,87, p<0,000)$, les plans suicidaires $(O R=48,43, p<0,000)$ et les tentatives suicidaires $(O R=30,04, p<0,000)$. Plus spécifiquement, les items sur l'automutilation par coupure $(O R=46,32, p<0,000)$, brûlure $(O R=16,2, p<0,000)$, empoisonnement $(O R=30,19, p<0.000)$ et par coups sur la tête $(O R=19,98$, $p<0,000)$ ont tous été démontrés significativement différents entre les deux groupes. L'anorexie $(O R=12,7, p<0,000)$, la boulimie $(p<0,000)$ et les problèmes de l'image de soi $(O R=15,74$, $p<0,000)$ ont été observés à travers les items de la perturbation de l'image de soi. Les items sur les 
caractéristiques psychotiques réunissaient les hallucinations $(p<0,000)$, les idées délirantes $(O R=17,43, p<0,000)$, la paranoïa $(O R=12,59, p<0,000)$ et les idées étranges $(O R=55,33$, $p<0,000)$. Au niveau des difficultés interpersonnelles, le groupe TPL a manifesté des résultats plus élevés aux items suivants : promiscuité $(O R=13,06, p<0,000)$, bagarre $(O R=3,76, p<0,000)$ et difficulté à se faire des amis $(O R=5,71, p<0,000)$. Finalement, les items de la labilité affective observaient l'humeur maussade $(O R=7,26, p<0,000)$, la sensibilité $(O R=8,73, p<0,000)$, le tempérament $(O R=7,56, p<0,000)$, le sentiment de vide ( $O R=32,37, p<0,000)$, l'ennui $(O R=9,32$, $p<0,000)$, la colère $(O R=3,99, p<0,000)$ et l'explosion affective $(O R=13,05, p<0,000)$.

En 2014, Ha, Balderas, Zanarini, Oldham et Sharp se sont penchés sur la comorbidité psychiatrique chez les adolescents ayant un diagnostic de TPL en comparaison avec une population psychiatrique adolescente sans TPL. Les mesures ont été colligées par les adolescents et les parents à l'aide de questionnaires auto-rapportés. Le Childhood Interview for DSM-IV Borderline Personality Disorder (CI-BPD) fut utilisé afin d'obtenir le diagnostic de TPL (approche catégorielle et dimensionnelle), ainsi que le Global Adaptive Functioning (GAF) pour le niveau de fonctionnement global. Le National Institute of Mental Health Diagnostic Interview Schedule for Children (NIMH DISC-IV) et le Car, Relax, Alone, Forget, Friends, Trouble (CRAFFT) ont mesuré les diagnostics psychiatriques et le Child Behavior Checklist (CBCL) et le YSR ont été utilisés pour mesurer les symptômes des problèmes psychiatriques. Tout d'abord, les adolescents ayant un TPL ont eu des résultats plus faibles au GAF $\left(t_{330}=4,16, p<0,001\right)$. Les résultats du CIBPD entre les deux groupes ont tous été démontrés statistiquement significatifs. Plusieurs différences concernant la présence d'une comorbidité avec d'autres troubles psychiatriques ont été observées entre le groupe contrôle et le groupe TPL. Selon les questionnaires auto-rapportés complétés par les adolescents avec une approche catégorielle, les troubles de l'humeur ( $x^{2}=27,4$, 
$p<0,001)$, le trouble de dépression majeur $\left(x^{2}=18,08, p<0,001\right)$, les troubles anxieux $\left(x^{2}=13,53\right.$, $p<0,001)$, le trouble obsessif compulsif $\left(x^{2}=14,97, p<0,001\right)$, le syndrome de stress posttraumatique $\left(x^{2}=9,26, p<0,002\right)$, le trouble d'anxiété de séparation $\left(x^{2}=10,7, p<0,001\right)$, la phobie spécifique $\left(x^{2}=17,67, p<0,001\right)$, les troubles externalisés $\left(x^{2}=19,02, p<0,001\right)$, le trouble d'opposition avec provocation $\left(x^{2}=31,47, p<0,001\right)$ et les troubles de conduite $\left(x^{2}=17,72, p<0,001\right)$ ont tous été rapportés significativement plus souvent chez les adolescents ayant un TPL. Aucune différence statistiquement significative n'a été observée au niveau de la dysthymie, des troubles de l'alimentation et du déficit de l'attention avec hyperactivité. Aucune différence significative n'a été rapportée par les parents en ce qui a trait aux troubles de l'axe I mentionnés. Cependant, les chercheurs ont observé les différences des symptômes psychiatriques de l'axe I entre les deux groupes avec une approche dimensionnelle. Les résultats ont changé drastiquement. Ainsi, selon les questionnaires rapportés par les adolescents, les symptômes des troubles internalisés $\left(t_{329}=-6,63, p<0,001\right)$, externalisés $\left(t_{329}=-7,14, p<0,001\right)$, affectifs $\left(t_{329}=-6,61, p<0,001\right)$ et anxieux $\left(t_{32}=-4,12, p<0,001\right)$ étaient significativement plus présents chez les adolescents ayant un TPL que ceux du groupe contrôle. De plus, le déficit de l'attention avec hyperactivité $\left(t_{32}=-6,95\right.$, $p<0,001)$, le trouble d'opposition avec provocation $\left(t_{329}=-5,35, p<0,001\right)$ et les troubles de la conduite $\left(t_{32}=-6,64, p<0,001\right)$ étaient également plus présents chez le groupe TPL. Plusieurs différences ont été observées parmi les résultats des questionnaires remplis par les parents. Cette fois-ci, les troubles internalisés $\left(t_{329}=-3,42, p=0,001\right)$, les troubles externalisés $\left(t_{32}=-3,32\right.$, $p=0,001)$, les troubles affectifs $\left(t_{32}=-5,24, p<0,001\right)$, le déficit de l'attention avec hyperactivité $\left(t_{32}=-2,81, p=0,005\right)$ et le trouble d'opposition avec provocation $\left(t_{329}=-2,75, p=0,007\right)$ ont été rapportés plus souvent chez les sujets du groupe TPL que ceux du groupe contrôle. 
Horesh, Orbach, Gothelf, Efrati et Apter (2003) ont voulu observer les diverses formes des comportements suicidaires chez les adolescents ainsi que les rôles du désespoir, de la dépression, de la colère et de l'impulsivité. Pour ce faire, les chercheurs ont comparé quatre groupes d'adolescents: avec TPL non suicidaire, avec TPL suicidaire, avec dépression majeure (DM) non suicidaire et avec DM suicidaire. Plusieurs outils ont été utilisés : le Child Suicide Potential Scale (CSPS) pour le potentiel suicidaire, le BDI pour la dépression, le Beck Hopelessness Scale (BHS) pour le désespoir, le Multidimensional Anger Inventory (MAI) pour la colère, le Overt Agression Scale (OAS) pour l'agressivité, le Impulsiveness-Control Scale (ICS) pour l'impulsivité et le Suicide Intent Scale (SIS) pour les intentions suicidaires. Le TPL fut diagnostiqué à l'aide du Child and Adolescent Version of the Affective Disorders and Schizophrenia (K-SADS-PI) et du DIB-R.

Les chercheurs ont d'abord comparé le groupe TPL avec le groupe DM sur les affects. Le groupe TPL présentait des résultats significativement plus élevés sur la colère $(F(1,63)=17,3$, $p<0,001)$, l'agression $(F(1,63)=25,3, p<0,001)$ et l'impulsivité $(F(1,63)=33,7, p<0,001)$. Aucune différence n'a été observée sur la dépression et le désespoir. Les chercheurs ont procédé à la même analyse en comparant le groupe suicidaire avec le groupe non suicidaire. Les adolescents suicidaires avaient des résultats plus élevés sur l'agression $(F(1,63)=8,2, p<0,01)$, la dépression $(F(1,63)=32, p<0,001)$ et le désespoir $(F(1,63)=26,3, p<0,001)$ que le groupe non suicidaire. Une analyse sur les interactions a par la suite été réalisée. Le groupe adolescent TPL suicidaire se retrouvait significativement plus impulsif que les adolescents TPL non suicidaires. Cette interaction entre l'impulsivité, le suicide et le diagnostic n'a pas été observée entre les groupes d'adolescents avec DM non suicidaire et suicidaire. Aucune interaction n'a été observée avec les autres variables. Les chercheurs ont également comparé le type de colère mesuré par le MAI, soit la colère interne et la colère externe. Les adolescents non suicidaires présentaient ainsi davantage 
de colère externe que les adolescents suicidaires $(F(1,63)=4,64, p<0,05)$. Le groupe TPL présentait également des résultats plus élevés sur la colère externe que les adolescents avec DM $(F(1,63)=13,68, \mathrm{p}<0,01)$. Les chercheurs ont ensuite procédé à des corrélations de Pearson. La combinaison du diagnostic de TPL et des symptômes d'impulsivité $(r=0,4, p<0,05)$ et d'agression $(r=0,5, p<0,01)$ corrélaient positivement et significativement avec les comportements suicidaires. Cette corrélation n'était pas présente avec le diagnostic de DM. Autant chez les adolescents TPL et ceux avec DM, la présence de dépression (TPL : $r=0,63, p<0,001$; DM : $r=0,6, p<0,001$ ) et de désespoir (TPL : $r=0,54, p<0,01$; DM : $r=0,71, p<0,01$ ) corrélait positivement et significativement avec les comportements suicidaires. Finalement, les adolescents DM suicidaires présentaient des résultats significativement plus élevés sur l'intention suicidaire que les adolescents TPL suicidaires $(t(31)=2,79, p<0,01)$.

Récemment, Kramer et collaborateurs (2017) ont voulu étudier le fonctionnement psychosocial des adolescents avec TPL. Pour ce faire, ils les ont comparés à un groupe d'adolescents sans troubles psychiatriques à l'aide des instruments suivants: Structured Clinical Interview for DSM-IV Childhood Diagnoses (KID-SCID), DIB-R, CI-BPD, Adolescent Version of the Background Information Schedule (BIS-AV) et GAF. Les adolescents TPL ont présenté des résultats plus faibles au GAF que les adolescents sans diagnostics. Au niveau du fonctionnement interpersonnel, les adolescents sans troubles psychiatriques rapportaient une meilleure relation avec leur père $(70 \%$ contre $43,3 \%, p=0,003)$ et leur mère $(95 \%$ contre $62,5 \%, p<0,001)$. Les adolescents avec TPL rapportaient une plus forte tendance à sortir de la maison $(56,7 \%$ contre $26,7 \%, p=0,001)$, cependant, ils rapportaient davantage de temps passé à être seul (36,5\% contre $8,3 \%, p=0,001)$. Aucune différence significative n'a été observée dans la qualité de la relation avec les amis. Seuls trois items du fonctionnement vocationnel ont été démontrés significativement 
différents entre les deux groupes. Les adolescents non psychiatriques rapportaient un meilleur historique au travail et à l'école $(100 \%$ contre $66,4 \%, p=0,002)$ et une participation plus fréquente à l'école ou dans des organisations communautaires $(56,7 \%$ contre $31,7 \%, p=0,002)$. Finalement, les adolescents avec TPL se retrouvaient plus souvent suspendus ou expulsés à l'école que le groupe contrôle $(36,5 \%$ contre $8,3 \%, p<0,001)$. Les adolescents ayant un TPL rapportaient ainsi un moins bon fonctionnement relationnel et vocationnel que les adolescents sans troubles psychiatriques.

Pour leur part, Lawrence, Allen et Chanen (2011) se sont intéressés aux schémas mésadaptés chez les adolescents avec TPL. Le groupe contrôle regroupait des adolescents qui ne présentaient aucun trouble de l'axe I et aucune caractéristique du TPL ou du trouble de personnalité antisociale. Le Structured Clinical Interview for DSM-IV Axis I Disorders - Patient Edition (SCID I/P), le SCID-II et le Diagnostic Interview for Personality Disorders (DIPD-IV) ont été utilisés afin d'obtenir les diagnostics de TPL. Les problèmes d'abus de substance furent mesurés par le Alcohol, Smoking and Substance Involvement Screening Test (ASSIST V3.0). Finalement, le Young Schema Questionnaire - Short Form (YSQ-S2) a été utilisé pour mesurer la présence de schémas mésadaptés. Les chercheurs ont par la suite comparé les moyennes des résultats de chaque type de schéma mésadapté entre le groupe d'adolescents TPL et le groupe contrôle. Les schémas d'abandon $\left(M_{\text {diff }}=15,1, \mathrm{p}<0,003\right)$, de méfiance $\left(M_{\text {diff }}=11,1, \mathrm{p}<0,003\right)$, d'isolement social $\left(M_{\text {diff }}=10,2, \mathrm{p}<0,003\right)$, de honte $\left(M_{\text {diff }}=12,5, \mathrm{p}<0,003\right)$, de déprivation émotionnelle $\left(M_{\text {diff }}=9,2\right.$, $\mathrm{p}<0,003)$ et de soumission $\left(M_{d i f f}=9,2, \mathrm{p}<0,003\right)$ ont tous été significativement plus présents chez le groupe TPL. Les schémas d'échec $\left(M_{d i f f}=10,2, \mathrm{p}<0,003\right)$, d'incompétence $\left(M_{\text {diff }}=9,3, \mathrm{p}<0,003\right)$, de vulnérabilité $\left(M_{\text {diff }}=7,7, \mathrm{p}<0,003\right)$, d'inhibition émotionnelle $\left(M_{\text {diff }}=5,5, \mathrm{p}<0,003\right)$ et du contrôle de soi insuffisant $\left(M_{d i f f}=8,4, \mathrm{p}<0,003\right)$ étaient également plus présent chez le groupe TPL. 
Cependant, aucune différence statistiquement significative n'a été retrouvée parmi les schémas d'enchevêtrement, du sacrifice de soi, des standards inflexibles et du sentiment d'entitlement $^{1}$. Les chercheurs ont ensuite observé la relation entre chaque critère diagnostique du TPL et chaque schéma maladaptif mentionné plus tôt. Le critère de la colère intense et inappropriée était négativement et significativement corrélé au schéma de la honte $(r=-0,37, \mathrm{p}<0,05)$, celui des efforts répétés afin d'éviter un abandon réel ou imaginaire était corrélé positivement au schéma d'abandon $(r=0,39, \mathrm{p}<0,05)$ et le critère d'idéation/dissociation paranoïaque relié au stress était positivement corrélé à trois schémas : déprivation émotionnelle $(r=0,43, \mathrm{p}<0,05)$, standards inflexibles $(r=0,45, \mathrm{p}<0,05)$ et inhibition émotionnelle $(r=0,45, \mathrm{p}<0,05)$. Plusieurs schémas mésadaptés sont ainsi présents chez la majorité des adolescents ayant un TPL. Cependant, cette étude met en évidence l'hétérogénéité de la symptomatologie de ce trouble ainsi que le lien entre certains critères diagnostiques et certains schémas internes spécifiques.

En 2012, Loas, Speranza, Pham-Scottez, Perez-Diaz et Corcos se sont penchés sur la relation entre l'alexithymie et le trouble de personnalité limite à l'adolescence. Ils ont ainsi comparé un groupe d'adolescents diagnostiqués avec TPL et un groupe contrôle non psychiatrique. Les troubles de l'axe I du DSM-IV ont été mesurés à l'aide du Schedule for Affective Disorders and Schizophrenia for School-Aged Children (K-SADS) et ceux de l'axe II avec la version française du Structured Interview for DSM-IV Personality (SIDP-IV). Les mesures de l'alexithymie, la dépression et l'anxiété furent obtenues à l'aide du Twenty-item Toronto Alexithymia Scale (TAS-20), du Second Version of the Beck Depression Inventory (BDI-II) et du State-Trait Anxiety Inventory (STAI-T). Tout d'abord, le trouble de dépression majeure était présent chez $35,6 \%$ des adolescents du groupe TPL et complètement absent chez le groupe contrôle. De plus, les chercheurs ont observé une différence statistiquement significative

${ }^{1}$ Entitlement: sentiment chez un individu que tout lui revient de droit, arrogance, enfant gâté 
concernant la prévalence de l'alexithymie entre les deux groupes $\left(x^{2}=22,5, d f=1, p<0,001\right)$. Le groupe TPL a également présenté des résultats plus élevés au TAS-20 que le groupe contrôle $(t(138)=6,1, p<0,001)$. Par la suite, les chercheurs ont procédé à deux ANCOVA pour observer la relation entre l'alexithymie et le TPL en contrôlant pour l'anxiété et la dépression. Utilisant le TAS-20 comme variable indépendante, le BDI-II comme covariable et la comparaison entre le groupe contrôle et le groupe TPL comme variable indépendante, l'effet de groupe n'était plus significatif. Cependant, l'effet de la covariable était significatif. Les mêmes conclusions furent observées lorsque la covariable était les résultats au STAI-T. Ainsi, la différence significative entre le groupe contrôle et le groupe TPL disparaissait lorsque la relation était contrôlée autant au niveau de la dépression que de l'anxiété, renforçant la théorie que l'alexithymie ne soit qu'un symptôme secondaire à la présence de dépression ou d'anxiété chez les adolescents ayant un TPL. Tel que mentionné plus tôt, la dépression majeure étant présente dans l'échantillon du groupe TPL, le niveau d'alexithymie pourrait être un symptôme indirect provenant plutôt de l'état dépressif chez ces adolescents.

Alors que le DIB fut originellement conçu pour faire le diagnostic du TPL chez une population adulte, McManus, Lerner, Robbins et Barbour (1984) se sont intéressés à la fiabilité et à la validité de ce test chez la population adolescente. Ils ont ainsi évalué deux groupes d'adolescents (avec TPL et contrôle psychiatrique sans TPL) afin d'observer si le test diagnostique pouvait être utilisé chez cette population. Les chercheurs ont procédé au diagnostic des adolescents à l'aide du Schedule for Affective Disorders and Schizophrenia (SADS) et du DIB. Ce qui nous intéresse particulièrement dans cette étude est la section où les chercheurs ont identifié spécifiquement les items du DIB ayant la capacité de différencier les adolescents TPL du groupe psychiatrique sans TPL. Tout d'abord, le résultat total au DIB permettait effectivement, selon 
l'étude, de différencier le groupe TPL du groupe contrôle. Les adolescents ayant un diagnostic de TPL avaient des résultats plus élevés au DIB ( 7,6 contre 4,4, $t=5,3172, p=0,0001)$. De plus, les sous-sections de l'impulsivité $(t=3,8561, p=0,0004)$, de l'affect $(t=4,5271, p=0,0001)$ et des relations interpersonnelles $(t=4,9149, p=0,0001)$ permettaient également de différencier les deux groupes. La sous-section de l'adaptation sociale pouvait seulement différencier les adolescents avec un TPL des adolescents schizophréniques, alors que la sous-section de la psychose ne pouvait différencier aucun groupe. Plus spécifiquement, 11 items du DIB différenciaient le groupe TPL du groupe psychiatrique contrôle. Dans la sous-section de l'impulsivité, l'automutilation, l'abus de drogue et les comportements antisociaux étaient plus fréquemment endossés par le groupe TPL. La colère, l'entitlement et la dysphorie chronique de la sous-section de l'affect étaient également plus présents chez les adolescents TPL. Les items de «n'être jamais seul, les conflits concernant la dépendance, les relations intenses et instables, l'hostilité et la fragmentation de la sous-section des relations interpersonnelles » différenciaient significativement les deux groupes. Finalement, seul l'item d'une vie sociale active de la sous-section de l'adaptation sociale permettait de faire la différence entre les deux groupes, le groupe psychiatrique contrôle ayant eu des résultats plus élevés. Ainsi, seuls les éléments de l'affect, de l'impulsivité et des relations interpersonnelles permettaient de bien différencier les adolescents TPL des adolescents psychiatriques contrôle, alors qu'aucune différence n'était présente concernant l'adaptation sociale et la psychose.

La dépression majeure étant un trouble étant fréquemment comorbide avec un diagnostic de TPL à l'adolescence, Pinto, Grapentine, Francis et Picariello (1996) se sont penchés sur les caractéristiques affectives et cognitives du TPL tout en contrôlant les effets de la dépression. Pour ce faire, ils ont comparé un groupe d'adolescents ayant un diagnostic de TPL et de DM et un groupe n'ayant été diagnostiqué qu'avec DM. Le diagnostic de TPL fut obtenu grâce au DIB-R, 
alors que celui de la dépression et du niveau de sévérité de cette dépression furent mesurés par le Revised Diagnostic Interview for Children and Adolescents (DICA-R-A) et le BDI. Au niveau des caractéristiques affectives, le Revised Children's Manifest Anxiety Scale (RCMSA) et le StateTrait Anger Expression Inventory (STAXI) ont été utilisés pour observer l'anxiété et la colère. Finalement, en ce qui a trait aux biais cognitifs, le Hopelessness Scale for Children (HSC) (désespoir), le Nowicki-Strickland Locus of Control Scale (LOC) (lieu de contrôle), le Children's Attributional Style Questionnaire (CASQ) (style attributif) et le Piers-Harris Children's SelfConcept Scale (PHCSCS) (concept de soi) ont été utilisés. Tout d'abord, autant les résultats du groupe TPL avec DM que ceux du groupe DM seulement démontraient un niveau modéré de sévérité sur la dépression, sans différences significatives. La sévérité des symptômes dépressifs n'a ainsi pas influencé les résultats suivants. Comparé aux moyennes de la population normative, autant le groupe TPL avec DM que le groupe DM seulement ont eu des résultats plus élevés sur les items du désespoir, de la colère, de l'anxiété, du style d'attribution négatif, du lieu de contrôle externe et d'un concept de soi pauvre. Cependant, le groupe TPL avec DM avaient des résultats plus élevés que le groupe contrôle sans être significativement différents. Une seule différence entre les deux groupes a émergé sur la mesure du PHCSCS (concept de soi). Le groupe avec TPL et DM ont démontré des résultats plus faibles sur cette mesure que le groupe sans TPL $(t(38)=-2,6$, $p<0,005)$. Les chercheurs se sont penchés sur les sections spécifiques afin de déterminer quelles dimensions du concept de soi étaient significativement différentes entre les deux groupes. Le groupe sans TPL avaient des résultats plus élevés dans les domaines de la satisfaction $(t(38)=-2,7$, $p<0,001)$, l'apparence physique $(t(38)=-2,29, p<0,01)$ et la popularité $(t(38)=-2,87, p<0,003)$. En contrôlant pour le trouble de dépression majeure, les chercheurs ont pu explorer la différence dans les symptômes affectifs et cognitifs entre le TPL et la DM. Seuls certains domaines du concept de 
soi ont été démontrés significativement différents chez ces deux groupes d'adolescents, alors que les symptômes affectifs, le désespoir et le style attributionnel ne permettaient pas de les différencier.

Plus récemment, Quek et collaborateurs (2018) ont poursuivi l'étude à propos de la capacité de mentalisation chez les adolescents avec TPL. Ils ont utilisé le Reflective Function Questionnaire for Youth (RFQY), le Reading the Mind in the Eyes Test (RMET) et le Movie for the Assessment of Social Cognition (MASC) pour comparer un groupe avec TPL et un groupe contrôle non psychiatrisé. Les diagnostics de TPL et des difficultés émotionnelles et comportementales furent réalisés à l'aide du Borderline Personality Features Scale for Children (BPFSC), du CI-BPD et du Strenghs and Difficulties Questionnaire (SDQ). Tout d'abord, la différence des résultats au RFQY était très grande et significative entre les groupes. Les adolescents ayant un TPL démontraient des résultats plus faibles que le groupe contrôle $(F(1,47)=31,78, p<0,01)$. Le groupe TPL avait également des résultats plus faibles au MASC $(F(1,47)=5,85, p<0,05)$. Plus spécifiquement, les chercheurs se sont penchés sur le type d'erreur de mentalisation rendant les résultats au MASC significativement différents. L'hypermentalisation était la seule erreur significativement différente entre les deux groupes $(F(1,47)=9,45, p<0,01)$, alors qu'il n'y en avait pas sur les items de la sous-mentalisation et de l'absence de mentalisation. En comparaison avec les adolescents sans trouble psychiatrique, ceux ayant un diagnostic de TPL présentaient un patron d'erreurs de mentalisation. Par le passé, la communauté scientifique mentionnait l'absence de la capacité de mentalisation chez les adolescents ayant un TPL. Cependant, Quek et collaborateurs (2018) ont pu déterminer que l'erreur de mentalisation la plus fréquente chez cette population est l'hypermentalisation. 
Par ailleurs, Serman, Johnson, Geller, Kanos et Zacharapoulou (2002) ont exploré la relation entre les symptômes de différents troubles de personnalité et l'abus de substance chez les adolescents. Les diagnostics ont été réalisés avec le SCID-II et les comportements d'abus de substances avec l'Adolescent Health Behavior Survey. En comparant chaque trouble de personnalité à l'usage de substance, les chercheurs ont observé que les adolescents ayant un TPL ou un trouble de personnalité antisocial rapportaient davantage de comportements reliés à l'abus de substance que les adolescents ayant d'autres troubles de personnalité. Ainsi, un plus grand nombre d'adolescents avec TPL mentionnaient avoir fumé la cigarette durant la dernière année $\left(x^{2}=3,92, p<0,05\right)$ en comparaison avec tous les autres adolescents sans TPL. Le même résultat fut observé concernant la consommation d'au moins cinq breuvages alcoolisé ou plus au courant des 30 derniers jours $\left(x^{2}=5,27, p<0,05\right)$. Aucune différence significative ne fut rapportée au sujet de l'usage quotidien de la cigarette ni de l'usage à l'année de l'alcool ou de drogues illicites. Ainsi, cette étude démontre qu'un certain patron d'abus de substances est présent chez les adolescents ayant un TPL pouvant les différencier des adolescents ayant d'autres troubles de personnalité, soit la consommation de cigarette au cours d'une année et la consommation d'alcool durant un mois.

La relation entre le suicide et le TPL à l'adolescence est couramment abordée par la communauté scientifique. Venta, Ross, Schatte et Sharp (2012) se sont penchés spécifiquement sur ce lien. Le CI-BPD et le BPFSC furent utilisés pour évaluer le TPL chez les adolescents. Les symptômes dépressifs furent mesurés par le YSR. Finalement, les comportements et les idées automutilatoires furent évalués par le Self-Injurious Thoughts and Behaviours Interview (SITBI), le Modified Scale for Suicide Ideation (MSSI) et le SIS. Tout d'abord, les chercheurs ont comparé le groupe TPL au groupe psychiatrique non TPL sur les idéations suicidaires. Le groupe TPL rapportait des idéations suicidaires significativement plus fréquentes en bas âge que le groupe 
contrôle $(t=2,76, p=0,007)$, ainsi qu'une fréquence plus haute $(t=-3,18, p=0,009)$ et une intensité plus forte $(t=-2,4, p=0,025)$ concernant ces idéations. Trois régressions linéaires ont été appliquées afin de contrôler pour la dépression. La relation entre le TPL et l'âge de début des idéations suicidaires $(\beta=-0,25, t=-2,34, p=0,022)$ et celle entre le TPL et la fréquence $(\beta=0,441, t=4,977$, $p<0,001)$ de ces idéations sont demeurées significatives. La relation entre le TPL et l'intensité des idéations suicidaires est disparue lorsqu'elle fut contrôlée pour la dépression. De plus, la différence entre le groupe TPL et le groupe psychiatrique contrôle sur l'idéation suicidaire (mesure continue) était statistiquement significative $(t=-3,7, p<0,001)$, le groupe TPL rapportant davantage d'idéations suicidaires. La relation entre le TPL et les idéations suicidaires, contrôlée pour la dépression, demeurait significative $(\beta=0,328, t=2,86, p=0,005)$. Par la suite, les chercheurs ont évalué les différences entre les deux groupes concernant les tentatives de suicide. Aucune différence statistiquement significative ne fut retrouvée quant à l'âge du début des tentatives, la fréquence de celles-ci et l'intention globale de mourir. Ainsi, seul le domaine des idéations suicidaires permettait la différenciation du groupe TPL du groupe psychiatrique sans TPL. Les adolescents rapportaient des idéations suicidaires plus tôt à l'adolescence, une fréquence plus forte de ces idéations et une intensité plus forte de celles-ci. Cependant, les caractéristiques des tentatives suicidaires ne permettaient pas de différencier les deux groupes.

De leur côté, Yen, Gagnon et Spirito (2013) ont observé l'historique et les caractéristiques des comportements suicidaires, ainsi que la capacité de réguler les affects, l'agression et la comorbidité des troubles de l'axe I chez les adolescents avec et sans trouble de personnalité limite. Les diagnostics du TPL et des troubles de l'axe I furent mesurés par le K-SADS-PL et le CI-BPD. Le Beck Scale for Suicide Ideation (BSS) et le Suicide Ideation Questionnaire (SIQ) furent utilisés pour observer les idéations suicidaires et le Functional Assessment of Self-Mutilation (FASM) pour 
les comportements automutilatoires. Le domaine des affects a été mesuré à l'aide du Negative Affective Self-Statement Questionnaire (NASSQ) et de l'Affect Intensity Measure (AIM). Finalement, l'agressivité fut mesurée avec le Aggression Questionnaire (AQ) et la régulation émotionnelle avec le Emotion Regulation Checklist - Adapted (ERC). Les trois critères les plus souvent rapportés parmi le groupe TPL étaient les comportements automutilatoires $(91,7 \%)$, l'impulsivité $(85,4 \%)$ et l'instabilité affective $(85,4 \%)$. Cependant, les plus grandes différences entre les deux groupes se retrouvaient sur les critères de la perturbation relationnelle $(66,7 \% \mathrm{TPL}$ contre $5,6 \%$ non TPL) et la colère $(89,6 \%$ TPL contre $23,6 \%$ non TPL). Les chercheurs se sont penchés sur les caractéristiques des comportements automutilatoires et suicidaires. Ainsi, le groupe TPL rapportait un plus haut taux de tentatives suicidaires avant une admission à un hôpital psychiatrique $\left(x^{2}=4,26, p=0,04\right)$. De plus, ce groupe se démarquait significativement du groupe psychiatrique non TPL relativement à un historique de tentatives suicidaires $\left(x^{2}=10,92, p=0,001\right)$. Cependant, aucune différence statistiquement significative ne fut observée concernant l'intention des tentatives suicidaires ni de la létalité de celles-ci. Aucune différence ne fut trouvée au sujet de la prévalence des comportements automutilatoires ou de la fréquence de ceux-ci. Finalement, l'intensité de l'idéation suicidaire ne permettait pas de différencier les deux groupes. Quelques différences furent observées concernant les troubles de l'axe I. Ainsi, le trouble de dépression majeur $\left(x^{2}=7,15, p<0,01\right)$, le trouble bipolaire $\left(x^{2}=5,4, p<0,05\right)$, les troubles du comportement perturbateur $\left(x^{2}=4,769, p<0,05\right)$, et les troubles anxieux $\left(x^{2}=7,057, p<0,01\right)$ étaient rapportés davantage par le groupe TPL. Lorsque les chercheurs ont utilisé les résultats dimensionnels, une nouvelle différence significative fut observée au sujet des troubles de l'alimentation $(r=0,22$, $p=0,02)$. 
Finalement, autant à l'aide des résultats catégoriques que dimensionnels, les résultats au GAF étaient significativement plus faibles chez le groupe TPL que le groupe psychiatrique contrôle. Quant au niveau d'agressivité, mesuré par le AQ, les résultats étaient significativement corrélés au TPL $(t=-2,21, p=0,03)$. Plus spécifiquement, la colère $(t=-2,3, p=0,024)$, l'hostilité $(t=-2,18, p=0,032)$ et l'agressivité indirecte $(t=-2,02, p=0,046)$ étaient les trois sous-sections produisant cette corrélation significative. Pour conclure, les résultats du AIM ne permettaient pas de différencier significativement le groupe TPL du groupe psychiatrique contrôle. De plus, seul le domaine de la labilité négative du ERC était significativement différent entre les deux groupes $(t=-2,54, p=0,013)$. Finalement, les résultats du groupe TPL relativement à la sous-catégorie de l'anxiété du NASSQ étaient significativement différents au groupe contrôle $(t=-2,18, p=0,03)$. Ainsi, les adolescents ayant un TPL rapportaient davantage de tentatives suicidaires et d'historique de tentatives de suicide avant l'admission à un hôpital psychiatrique que le groupe sans TPL. De plus, le trouble de dépression majeure, le trouble bipolaire, les troubles du comportement perturbateur, les troubles anxieux et les troubles de l'alimentation étaient plus comorbides chez les adolescents avec TPL que le groupe psychiatrique contrôle. Finalement, la colère, l'hostilité et l'agressivité indirecte, ainsi que la labilité négative et l'anxiété étaient des caractéristiques significativement plus souvent présentes chez le groupe TPL.

L'étude de Zanarini, Temes, Magni, Fitzmaurice, Aguirre et Goodman (2017) est particulièrement intéressante, puisque les chercheurs ont tenté d'observer les symptômes du TPL chez des adolescents non psychiatriques, des adolescents ayant un TPL et des adultes ayant ce diagnostic. Le Structured Clinical Interview for DSM-III-R Axis I Disorders (SCID) (pour les troubles de l'axe I et les troubles de personnalité) et le DIB-R ont été utilisés chez le groupe adulte ayant un TPL. Le KID-SCID, le DIB-R et le CI-BPD ont été utilisés pour les deux groupes 
adolescents avec et sans TPL. Tout d'abord, les adolescents ayant un TPL ont démontrés des résultats plus faibles au GAF que les adolescents sans TPL $\left(x^{2}=42,34, \mathrm{p}<0,001\right)$ et les adultes avec TPL $\left(x^{2}=4,6, \mathrm{p}<0,001\right)$. Ensuite, les chercheurs ont observé les différences entre les trois groupes sur 24 symptômes répartis en cinq catégories: affects, cognition, impulsivité, relations interpersonnelles et symptômes du TPL. Des différences statistiquement significatives furent observées sur toutes les caractéristiques affectives entre le groupe adolescent TPL et le groupe adolescent non psychiatrique: dépression majeure $(O R=109,39, p<0,001)$, désespoir ou culpabilité $(O R=96,05, p<0,001)$, colère $(O R=17,87, p<0,001)$, anxiété $(O R=19,14, p<0,001)$ et sentiment de vide $(O R=83,28, p<0,001)$. Aucune caractéristique n'a permis de différencier le groupe adolescent TPL du groupe adulte TPL.

Au niveau des caractéristiques cognitives, les pensées étranges $(O R=17,19, p<0,001)$, la paranoïa non délirante $(O R=28,6, p<0,001)$ et les pensées quasi psychotiques $(O R=26,38$, $p<0,001)$ étaient davantage rapportées par le groupe adolescent TPL que le groupe adolescent non psychiatrique. Les pensées quasi psychotiques étaient également observées plus souvent chez le groupe adulte TPL que le groupe adolescent TPL $(O R=2,97, p<0,001)$. De plus, l'abus de substance $(O R=18,3, p<0,001)$, la déviance sexuelle $(O R=19,85, p<0,001)$, l'automutilation $(O R=156,26, p<0,001)$ et le suicide manipulatif $(O R=249,84, p<0,001)$, de la catégorie impulsivité, étaient présents davantage chez les adolescents TPL que les adolescents non psychiatriques. Aucune différence significative n'était présente entre le groupe adolescent TPL et le groupe adulte TPL. Relativement aux caractéristiques des relations interpersonnelles, l'intolérance à la solitude $(O R=31,94, \quad p<0,001)$, les préoccupations concernant l'abandon/annihilation $(O R=45,46, p<0,001)$, la contre-dépendance $(O R=40,39, p<0,001)$, les relations houleuses $(O R=29,96, p<0,001)$, la dépendance/masochisme $(O R=7,56, p<0,001)$, la 
dévaluation/manipulation $(O R=5,14, p<0,001)$ et l'entitlement/exigence $(O R=11,84, p<0,001)$ étaient plus présents chez le groupe adolescent TPL que le groupe adolescent non psychiatrique. La dépendance/masochisme $(O R=4,86, p<0,001)$ et la dévaluation/manipulation $(O R=3,29$, $p<0,001)$ étaient également statistiquement plus présents chez le groupe adulte TPL que le groupe adolescent TPL. Aucune différence significative n'était présente concernant la régression durant la psychothérapie. Finalement, dans la catégorie des symptômes du TPL, l'instabilité affective $(O R=189,76, p<0,001)$ et la perturbation identitaire $(O R=26,8, p<0,001)$ étaient plus souvent présentes chez le groupe adolescent TPL que le groupe adolescent non psychiatrique. Ainsi, de nombreuses différences furent observées entre les adolescents ayant un TPL et les adolescents non psychiatriques, spécifiquement au sujet de la dépression majeure, du désespoir, du sentiment de vide, de l'automutilation, du suicide manipulatif et de l'instabilité affective. Les chercheurs ont également pu observer un patron symptomatique significativement différent entre les adolescents ayant un TPL et les adultes avec ce diagnostic. Les adultes rapportaient plus souvent des pensées quasi psychotiques, la dépendance, la dévaluation/manipulation et des problèmes de contretransfert au courant de la psychothérapie ou un sentiment de traitement spécial dans les relations interpersonnelles. 
Tableau III - Caractéristiques des études

\begin{tabular}{|c|c|c|c|c|}
\hline \multirow{2}{*}{ Études } & \multicolumn{3}{|c|}{ Échantillons (n (\% sexe féminin); âge) } & \multirow[t]{2}{*}{ Éléments comparés } \\
\hline & TPL & $\begin{array}{l}\text { Autre trouble } \\
\text { psychiatrique }\end{array}$ & $\begin{array}{l}\text { Sans trouble } \\
\text { psychiatrique }\end{array}$ & \\
\hline $\begin{array}{l}\text { Atlas, J. A. } \\
\text { (1996) }\end{array}$ & $\begin{array}{c}26(100 \%) \\
M=14,80\end{array}$ & $\begin{array}{l}12(100 \%) \\
M=14,80\end{array}$ & - & Dépression et caractéristiques dissociatives \\
\hline $\begin{array}{l}\text { Chabrol et } \\
\text { al. (2002) }\end{array}$ & $\begin{array}{c}24(83,3 \%) \\
M=16,70\end{array}$ & - & $\begin{array}{l}94(62,8 \%) \\
M=16,70\end{array}$ & $\begin{array}{l}\text { Facteurs comprenant quatre sous-sections : affects, } \\
\text { cognition, impulsivité et relations interpersonnelles }\end{array}$ \\
\hline $\begin{array}{l}\text { Chanen et } \\
\text { al. (2007) }\end{array}$ & $\begin{array}{c}46(68,4 \%) \\
M=16,24\end{array}$ & $\begin{array}{c}88(68,4 \%) \\
M=16,24\end{array}$ & $\begin{array}{c}43(68,4 \%) \\
M=16,24\end{array}$ & Fonctionnement psychosocial et psychopathologie \\
\hline $\begin{array}{l}\text { Goodman et } \\
\text { al. }(2010)\end{array}$ & $\begin{array}{l}321(100 \%) \\
M=24,04\end{array}$ & $\begin{array}{c}87(100 \%) \\
M=21,59\end{array}$ & - & $\begin{array}{l}\text { Affectivité, problèmes interpersonnels, impulsivité, } \\
\text { victimisation, automutilation, préoccupations relatives à } \\
\text { l'image de soi et caractéristiques psychotiques }\end{array}$ \\
\hline $\begin{array}{l}\text { Goodman et } \\
\text { al. (2013) }\end{array}$ & $\begin{array}{l}97(0 \%) \\
M=25,80\end{array}$ & $\begin{array}{l}166(0 \%) \\
M=25,70\end{array}$ & - & $\begin{array}{l}\text { Affectivité, problèmes interpersonnels, impulsivité, } \\
\text { victimisation, automutilation, préoccupations relatives à } \\
\text { l'image de soi et caractéristiques psychotiques }\end{array}$ \\
\hline $\begin{array}{l}\text { Ha et al. } \\
(2014)\end{array}$ & $\begin{array}{c}110(80 \%) \\
M=15,35\end{array}$ & $\begin{array}{l}225(52,9 \%) \\
\quad M=15,44\end{array}$ & - & $\begin{array}{l}\text { Symptomatologie limite, troubles de l'humeur, troubles } \\
\text { anxieux, troubles de l'alimentation et troubles externalisés }\end{array}$ \\
\hline $\begin{array}{l}\text { Horesh et al. } \\
\text { (2003) }\end{array}$ & $\begin{array}{c}33(78,8 \%) \\
M=15,00\end{array}$ & $\begin{array}{c}32(75,0 \%) \\
M=15,00\end{array}$ & - & $\begin{array}{l}\text { Comportement suicidaire, dépression, désespoir, colère, } \\
\text { agression, impulsivité, intention suicidaire }\end{array}$ \\
\hline $\begin{array}{l}\text { Kramer et } \\
\text { al. (2017) }\end{array}$ & $\begin{array}{l}104(94,2 \%) \\
M=15,70\end{array}$ & - & $\begin{array}{c}60(83,3 \%) \\
M=14,80\end{array}$ & Fonctionnement interpersonnel et professionnel \\
\hline $\begin{array}{l}\text { Lawrence et } \\
\text { al. (2011) }\end{array}$ & $\begin{array}{l}30(90 \%) \\
M=18,95\end{array}$ & - & $\begin{array}{l}28(67,9 \%) \\
\mathrm{M}=19,19\end{array}$ & Schémas inadaptés \\
\hline
\end{tabular}




\begin{tabular}{|c|c|c|c|c|}
\hline $\begin{array}{l}\text { Loas et al. } \\
(2012)\end{array}$ & $\begin{array}{l}85(87 \%) \\
M=16,30\end{array}$ & - & $\begin{array}{l}85(87 \%) \\
M=16,30\end{array}$ & Alexithymie \\
\hline $\begin{array}{l}\text { McManus et } \\
\text { al. (1984) }\end{array}$ & $\begin{array}{l}16(75 \%) \\
M=15,50\end{array}$ & $\begin{array}{l}32(50 \%) \\
M=15,30\end{array}$ & - & $\begin{array}{c}\text { Adaptation sociale, impulsivité, affects, psychose, relations } \\
\text { interpersonnelles }\end{array}$ \\
\hline $\begin{array}{l}\text { Pinto et al. } \\
\text { (1996) }\end{array}$ & $\begin{array}{c}19(100 \%) \\
M=14,81\end{array}$ & $\begin{array}{c}21(100 \%) \\
M=14,91\end{array}$ & - & $\begin{array}{l}\text { Anxiété, colère, désespoir, lieu de maîtrise, style } \\
\text { attributionnel et concept de soi }\end{array}$ \\
\hline $\begin{array}{l}\text { Quek et al. } \\
\text { (2018) }\end{array}$ & $\begin{array}{c}26(88,5 \%) \\
M=15,65\end{array}$ & - & $\begin{array}{l}25(80 \%) \\
M=15,12\end{array}$ & Capacités de mentalisation \\
\hline $\begin{array}{l}\text { Serman et } \\
\text { al. (2002) }\end{array}$ & $\begin{array}{c}15(93,3 \%) \\
M=16,30\end{array}$ & $\begin{array}{c}106(66 \%) \\
M=16,30\end{array}$ & - & Consommation de substances \\
\hline $\begin{array}{l}\text { Venta et al. } \\
(2012)\end{array}$ & $\begin{array}{c}14(71,4 \%) \\
M=14,63\end{array}$ & $\begin{array}{l}92(64,1 \%) \\
M=14,63\end{array}$ & - & Idéation suicidaire, tentative de suicide \\
\hline $\begin{array}{l}\text { Yen et al. } \\
\text { (2013) }\end{array}$ & $\begin{array}{l}47(80,9 \%) \\
\mathrm{M}=15,30\end{array}$ & $\begin{array}{l}72(58,3 \%) \\
M=15,30)\end{array}$ & - & $\begin{array}{l}\text { Comportements suicidaires, automutilation, troubles } \\
\text { psychiatriques et dysrégulation comportementale et } \\
\text { affective }\end{array}$ \\
\hline $\begin{array}{l}\text { Zanarini et } \\
\text { al. (2017) }\end{array}$ & $\begin{array}{l}104(94,2 \%) \\
\mathrm{M}=15,70\end{array}$ & - & $\begin{array}{l}60(83,3 \%) \\
M=14,80\end{array}$ & $\begin{array}{l}\text { Caractéristiques affectives, cognitives et interpersonnelles, } \\
\text { impulsivité, instabilité affective et trouble identitaire }\end{array}$ \\
\hline
\end{tabular}


Tableau IV - Méthodologie des études

\begin{tabular}{lccc}
\hline Études & $\begin{array}{c}\text { Taille } \\
\text { d'échantillon } \\
\text { total (N) }\end{array}$ & Outils d'évaluation utilisés & $\begin{array}{c}\text { Analyses statistiques } \\
\text { d'effet }\end{array}$ \\
\hline $\begin{array}{l}\text { Atlas, } \text { J. } \text { A. } \\
(1996)\end{array}$ & $\mathrm{N}=38$ & $\begin{array}{c}\text { Diagnostic Interview for Borderlines (DIB) } \\
\text { Beck Depression Inventory (BDI) }\end{array}$ & Test-t \\
& & Dissociative Experiences Scale Revised (DES-R) & - \\
\end{tabular}

\begin{tabular}{|c|c|c|c|}
\hline $\begin{array}{l}\text { Chabrol et } \\
\text { al. (2002) }\end{array}$ & $\mathrm{N}=118$ & Revised Diagnostic Interview for Borderlines (DIB-R) & $\begin{array}{l}\text { Test-t avec correction de } \\
\text { Bonferroni } \\
\text { Analyses factorielles : Scree } \\
\text { test et méthode varimax } \\
\text { ANOVA }\end{array}$ \\
\hline
\end{tabular}

Chanen et $\quad \mathrm{N}=177$
al. (2007)

Structured Clinical Interview for DSM-IV Axis 1 Disorders (SCID-I)

Kiddie Schedule for Affective Disorders and Schizophrenia - Present and Lifetime Version (K-SADS-PL)

Composite International Diagnostic Interview - Auto (CIDI-Auto)

Structured Clinical Interview for DSM-IV Axis II Disorders (SCID-II)

Youth Self-Report (YSR)

Young Adult Self-Report (YASR)

Health of the Nation Outcome Scales for Children and Adolescents (HoNOSCA)

Social and Occupational Functioning Assessment Scale

(SOFAS) 


$\begin{array}{lcc}\text { Goodman } & \mathrm{N}=408 & \begin{array}{c}\text { McLean Screening Instrument for Borderline } \\ \text { et al. (2010) }\end{array} \\ \text { Personality Disorder (MSI-BPD) }\end{array}$

Personality Disorder (MSI-BPD)
Chi-test avec correction de Holm-Bonferroni

Analyses de régression hiérarchiques

Analyse Wald Chi-Square

Statistique Nagelkerke

$\begin{array}{lcc}\text { Goodman } & \mathrm{N}=263 & \begin{array}{c}\text { McLean Screening Instrument for Borderline } \\ \text { et al. (2013) }\end{array} \\ \text { Personality Disorder (MSI-BPD) }\end{array}$

Chi-test avec correction de Holm-Bonferroni

Analyses de régression hiérarchiques

Analyse Wald Chi-Square

Statistique Nagelkerke
Modéréesélevées

\begin{tabular}{|c|c|c|c|c|}
\hline $\begin{array}{l}\text { Ha et al. } \\
(2014)\end{array}$ & $\mathrm{N}=335$ & $\begin{array}{c}\text { Childhood Interview for DSM-IV Borderline } \\
\text { Personality Disorder (CI-BPD) } \\
\text { Global Adaptive Functioning (GAF) } \\
\text { National Institute of Mental Health Diagnostic } \\
\text { Interview Schedule for Children (NIMH DISC-IV) } \\
\text { Car, Relax, Alone, Forget, Friends, Trouble (CRAFFT) } \\
\text { Child Behavior Checklist (CBCL) } \\
\text { Youth Self-Report (YSR) }\end{array}$ & $\begin{array}{l}\text { Chi-test avec correction de } \\
\text { Bonferroni } \\
\text { Régression linéaire } \\
\text { Analyses bivariées }\end{array}$ & $\begin{array}{l}\text { Faibles- } \\
\text { élevées }\end{array}$ \\
\hline $\begin{array}{l}\text { Horesh et } \\
\text { al. (2003) }\end{array}$ & $\mathrm{N}=65$ & $\begin{array}{c}\text { Child Suicide Potential Scale (CSPS) } \\
\text { Beck Depression Inventory (BDI) } \\
\text { Beck Hopelessness Scale (BHS) } \\
\text { Multidimensional Anger Inventory (MAI) }\end{array}$ & $\begin{array}{c}\text { MANOVA } \\
\text { Analyse des effets simples } \\
\text { pour les interactions }\end{array}$ & $\begin{array}{c}\text { Modérées- } \\
\text { élevées }\end{array}$ \\
\hline
\end{tabular}




\section{Horesh et \\ al. (2003)}

(suite)
Overt Aggression Scale (OAS)

Impulsiveness-Control Scale (ICS)

Suicide Intent Scale (SIS)
Coefficients de Pearson

pour les corrélations

Child and Adolescent Version of the Affective

Disorders and Schizophrenia (K-SADS-PI)

Revised Diagnostic Interview for Borderlines (DIB-R)

Test-t de Student pour les variables continues

Chi-test de Pearson pour les variables binaires

Régressions logistiques

Méthode de régression logistique de Firth

Global Adaptive Functioning (GAF)

$\begin{array}{lcc}\begin{array}{l}\text { Lawrence } \\ \text { et al. (2011) }\end{array} & \mathrm{N}=58 & \begin{array}{c}\text { Structured Clinical Interview for DSM-IV Axis I } \\ \text { Disorders }- \text { Patient Edition (SCID I/P) }\end{array} \\ \text { Structured Clinical Interview for DSM-IV Personality } \\ \text { Disorders (SCID-II) } \\ \text { Diagnostic Interview for Personality Disorders } \\ \text { (DIPD-IV) } \\ \text { Alcohol, Smoking and Substance Involvement } \\ \text { Screening Test (ASSIST V3.0) } \\ \text { Young Schema Questionnaire }- \text { Short Form (YSQ-S2) }\end{array}$

Comparaisons de moyennes Modérées avec correction de Bonferroni

Corrélations produitmoment de Pearson

$\begin{array}{lccc}\text { Loas et al. } & \mathrm{N}=170 & \begin{array}{c}\text { Schedule for Affective Disorders and Schizophrenia for } \\ \text { (2012) }\end{array} & \begin{array}{c}\text { Analyse factorielle } \\ \text { confirmatoire }\end{array}\end{array}$


Loas et al.
(2012)

(suite)
Structured Interview for DSM-IV Personality (SIDP-IV)

Twenty-item Toronto Alexithymia Scale (TAS-20)

Second Version of the Beck Depression Inventory

(BDI-II)

State-Trait Anxiety Inventory (STAI-T)
Alpha de Cronbach

Test-t de Student

Chi-test

ANOVA

\begin{tabular}{|c|c|c|c|}
\hline $\begin{array}{l}\text { McManus } \\
\text { et al. (1984) }\end{array}$ & $\mathrm{N}=48$ & $\begin{array}{c}\text { Schedule for Affective Disorders and Schizophrenia } \\
\text { (SADS) } \\
\text { Diagnostic Interview for Borderlines (DIB) }\end{array}$ & $\begin{array}{c}\text { Statistique Kappa } \\
\text { Corrélation produit-moment } \\
\text { de Pearson } \\
\text { Test exact de Fisher } \\
\text { ANOVA } \\
\text { Test-t }\end{array}$ \\
\hline
\end{tabular}

\begin{tabular}{|c|c|c|c|c|}
\hline $\begin{array}{l}\text { Pinto et al. } \\
\text { (1996) }\end{array}$ & $\mathrm{N}=40$ & $\begin{array}{c}\text { Revised Diagnostic Interview for Borderlines (DIB-R) } \\
\text { Revised Diagnostic Interview for Children and } \\
\text { Adolescents (DICA-R-A) } \\
\text { Beck Depression Inventory (BDI) } \\
\text { Revised Children's Manifest Anxiety Scale (RCMSA) } \\
\text { State-Traits Anger Expression Inventory (STAXI) } \\
\text { Hopelessness Scale for Children (HSC) } \\
\text { Nowicki-Strickland Locus of Control Scale (LOC) } \\
\text { Children's Attributional Style Questionnaire (CASQ) } \\
\text { Piers-Harris Children's Self-Concept Scale (PHCSCS) }\end{array}$ & Test-t & - \\
\hline $\begin{array}{l}\text { Quek et al. } \\
\text { (2018) }\end{array}$ & $\mathrm{N}=51$ & $\begin{array}{l}\text { Reflective Function Questionnaire for Youth (RFQY) } \\
\text { Reading the Mind in the Eyes Test (RMET) } \\
\text { Movie for the Assessment of Social Cognition (MASC) }\end{array}$ & $\begin{array}{l}\text { Analyses préliminaires } \\
\text { Test de Shapiro-Wilk } \\
\text { Test de Levene }\end{array}$ & $\begin{array}{l}\text { Modérée, } \\
\text { élevée et } \\
\text { très élevée }\end{array}$ \\
\hline
\end{tabular}


Quek et al.
(2018)

(suite)
Test-t

Chi-test avec correction de Yates

Test exact de Fisher ANCOVA
Serman et $\quad \mathrm{N}=121$ al. (2002)
Structured Clinical Interview for DSM-IV Personality Disorders (SCID-II)

Adolescent Health Behavior Survey
Chi-test avec correction de Bonferroni

Analyses de distribution Odd ratio

Analyses des tableaux de contingence

\begin{tabular}{|c|c|c|c|c|}
\hline $\begin{array}{l}\text { Venta et al. } \\
\text { (2012) }\end{array}$ & $\mathrm{N}=106$ & $\begin{array}{c}\text { Childhood Interview for DSM-IV Borderline } \\
\text { Personality Disorder (CI-BPD) } \\
\text { Borderline Personality Features Scale for Children } \\
\text { (BPFSC) } \\
\text { Youth Self-Report (YSR) } \\
\text { Self-Injurious Thoughts and Behaviours Interview } \\
\text { (SITBI) } \\
\text { Modified Scale for Suicide Ideation (MSSI) } \\
\text { Suicide Intent Scale (SIS) }\end{array}$ & $\begin{array}{c}\text { Test-t } \\
\text { Analyses de régressions } \\
\text { linéraires } \\
\text { Corrélations de Pearson }\end{array}$ & $\begin{array}{l}\text { Modérées } \\
\text { et élevées }\end{array}$ \\
\hline $\begin{array}{l}\text { Yen et al. } \\
\text { (2013) }\end{array}$ & $\mathrm{N}=119$ & $\begin{array}{c}\text { Kiddie Schedule for Affective Disorders and } \\
\text { Schizophrenia - Present and Lifetime Version } \\
\text { (K-SADS-PL) } \\
\text { Childhood Interview for DSM-IV Borderline } \\
\text { Personality Disorder (CI-BPD) }\end{array}$ & $\begin{array}{c}\text { T-test } \\
\text { Chi-test } \\
\text { Corrélations de Pearson } \\
\text { Corrélations de Spearman } \\
\text { Statistique Kappa }\end{array}$ & $\begin{array}{l}\text { Faibles et } \\
\text { modérées }\end{array}$ \\
\hline
\end{tabular}


Yen et al.
(2013)

(suite)
Beck Scale for Suicide Ideation (BSS)

Suicide Ideation Questionnaire (SIQ)

Functional Assessment of Self-Mutilation (FASM)

Negative Affective Self-Statement Questionnaire

$$
\text { (NASSQ) }
$$

Affect Intensity Measure (AIM)

Aggression Questionnaire (AQ)

Emotion Regulation Checklist - Adapted (ERC)
Zanarini et $\quad \mathrm{N}=164$ al. (2017)
Structured Clinical Interview for DSM-III-R Axis I Disorders (SCID)

Revised Diagnostic Interview for Borderlines (DIB-R)

Structured Clinical Interview for DSM-IV Childhood Diagnoses (KID-SCID)

Childhood Interview for DSM-IV Borderline

Personality Disorder (CI-BPD)

Global Adaptive Functioning (GAF)
Test-t de Student

Chi-test de Pearson

Régressions logistiques

Méthode de régression

logistique de Firth 


\section{Discussion}

Plusieurs symptômes et comorbidités ont été observés par les études mentionnées dans cette recension systématique. Certains résultats ont été mis en évidence plus régulièrement que d'autres. La recension des études a permis d'obtenir des patrons symptomatiques permettant de différencier les adolescents ayant un TPL de ceux n'ayant aucun trouble psychiatrique, ceux ayant un trouble psychiatrique autre que le TPL et ceux ayant un autre trouble de personnalité. Certaines études ont également permis d'observer un patron symptomatique précis au sujet du suicide chez les sujets avec un TPL.

Premièrement, les adolescents avec un diagnostic du TPL présentaient davantage de problèmes internalisés et externalisés que les adolescents non psychiatrisés. L'automutilation, la dépression majeure, l'instabilité affective, le désespoir, la colère, l'anxiété, le sentiment de vide, les tentatives et menaces suicidaires, l'intolérance à la solitude et la dépendance sont quelques symptômes internalisés observés. Quant aux problèmes externalisés, les études ont relevé l'impulsivité, les troubles d'abus de substances, les troubles alimentaires, la déviance sexuelle, les relations interpersonnelles houleuses et les difficultés scolaires (suspension et expulsion). Plusieurs problèmes psychotiques (pensées étranges, paranoïa non délirante et pensées quasi psychotiques) ont également été mentionnés, ainsi que la présence de schémas mésadaptés et des erreurs de mentalisation (hypermentalisation).

Deuxièmement, les études ont souligné plusieurs différences entre les sujets ayant un TPL et ceux ayant un autre diagnostic psychiatrique. La présence d'un mélange des troubles internalisés et externalisés était encore observée chez les sujets TPL. Les adolescents avec un TPL rapportaient plus régulièrement des symptômes d'impuissance, de dépression majeure, de solitude, de 
manipulation, d'entitlement, de troubles de l'humeur, de troubles anxieux, de phobie, de syndrome de stress post-traumatique, d'anxiété de séparation, d'automutilation, de comportements suicidaires, de colère et de trouble obsessif-compulsif. Ces symptômes étaient souvent comorbides avec des troubles externalisés: la déviance sexuelle, le trouble d'opposition avec provocation, les troubles de conduite, l'impulsivité, les troubles d'abus de substance, les comportements antisociaux, l'hostilité, les relations intenses et instables et l'agressivité indirecte. Les adolescents ayant un TPL avaient des résultats plus élevés sur la dysphorie et sur certains troubles psychotiques que les adolescents avec un autre trouble psychiatrique.

Troisièmement, certains symptômes permettaient de différencier les adolescents avec un TPL de ceux ayant un autre trouble de personnalité. Les troubles de l'humeur, les troubles de comportements, les troubles d'abus de substance, particulièrement avec la nicotine et l'alcool, l'absentéisme scolaire, les troubles internalisés et les troubles externalisés étaient rapportés plus souvent par les adolescents ayant un TPL. La combinaison de troubles internalisés et externalisés est, encore une fois, présente plus fréquemment chez les sujets ayant un TPL que chez les adolescents du groupe contrôle.

Dernièrement, plusieurs études s'étant penchées sur les caractéristiques des tentatives suicidaires chez les adolescents avec et sans TPL, un patron symptomatique a pu être observé. Ainsi, les adolescents ayant un TPL et étant suicidaires rapportaient davantage d'impulsivité que le groupe TPL non suicidaire. La combinaison du diagnostic de TPL et la présence d'agressivité et d'impulsivité corrélait positivement avec les comportements suicidaires. Néanmoins, l'intensité des intentions suicidaires était plus forte chez les adolescents suicidaires ayant un diagnostic de dépression majeure que chez les adolescents suicidaires avec TPL. Cependant, les idées suicidaires étaient plus fréquentes chez les adolescents avec un TPL et débutaient en plus bas âge. 
La présente recension systématique comporte certaines limites. Tout d'abord, afin d'avoir un portrait le plus global possible sur l'étude des symptômes associés au TPL durant l'adolescence, plusieurs études retenues ont utilisé des questionnaires afin de construire leur groupe TPL. Cependant, ces instruments, tels que le DIB et le MSI-BPD, n'ont pas été mis au point pour des fins diagnostiques. Ces questionnaires, fonctionnant avec un seuil de coupure, peuvent provoquer des faux positifs. Finalement, plusieurs études ne mentionnent pas l'ampleur des différences des résultats. Nous ne pouvons donc pas évaluer l'importance de leurs résultats significatifs.

Cependant, les résultats observés dans notre recension systématique sont appuyés par plusieurs autres études. Dans leur revue de la littérature, Chanen et Kaess (2012) ont constaté que l'abus de substance à l'adolescence, spécifiquement l'alcool, prédisait le diagnostic de TPL à l'âge adulte. Les adolescents ayant un TPL rapportaient un niveau plus élevé d'automutilation, ainsi que de comorbidité avec le trouble d'attention avec hyperactivité et le trouble oppositionnel avec provocation. Selon leurs observations, la présence du trouble de l'attention, du trouble de la régulation émotionnelle et des troubles de comportements prédisait certains symptômes du TPL à l'âge adulte. Pour leur part, Matusiewicz, Weaverling et Lejuez (2014) ont étudié la dysrégulation émotionnelle chez les adolescents ayant un TPL. Leurs résultats appuient les observations faites dans notre revue de la littérature au sujet de l'instabilité émotionnelle chez ces sujets. Selon les chercheurs, les adolescents avec un TPL avaient plus de difficultés à se réguler émotionnellement et possédaient moins de stratégies d'autorégulations. Leurs sujets rapportaient ainsi des réactions émotionnelles plus fortes en réponse à divers stresseurs. Plusieurs symptômes examinés dans notre recension systématique ont également été constatés en 1991 par Meijer et Treffers. Selon leur propre revue de la littérature, l'ennui, la difficulté à se concentrer, l'anxiété, les comportements antisociaux, les comportements passifs agressifs, le trouble obsessionnel compulsif, l'anorexie, 
l'anxiété de séparation, les symptômes schizoïdes et les tentatives suicidaires étaient des symptômes du TPL rapportés par leurs sujets à l'adolescence.

Une certaine tendance se dégage lorsqu'on examine la variété des symptômes rapportés au fil des ans au sujet des adolescents avec un TPL. La combinaison de symptômes internalisés (dépression, anxiété, automutilation) et de symptômes externalisés (impulsivité, abus de substances, difficultés relationnelles) revient constamment dans la majorité des études de notre revue de la littérature. En 2014, Ha et collaborateurs montrent bien ce phénomène et nomment cette combinaison de symptômes: complex comorbidity (comorbidité complexe). Selon eux, c'est précisément cette interaction de symptômes qui est associée à la symptomatologie du TPL. La présence de cette comorbidité complexe serait ainsi un bon indicateur de ce trouble à l'adolescence et permettrait un diagnostic et un traitement hâtifs. En d'autres mots, la présence d'impulsivité et de dysrégulation émotionnelle serait le mécanisme central du TPL (Fossati, Gratz, Maffei et Barroni, 2013).

La littérature scientifique au sujet du TPL à l'adolescence ne se limite pas aux symptômes mentionnés dans notre recension systématique. Plusieurs études examinent la différence symptomatique entre les garçons et les filles, démontrant la présence d'une hétérogénéité à ce niveau (Bradley, Conklin et Westen, 2005). Huynh, Guilé, Breton et Godbout (2016) ont exploré le cycle sommeil-éveil chez les adolescents avec TPL en comparaison avec des adolescents avec un trouble bipolaire et des adolescents non psychiatrisés, observant des différences à ce sujet. Pour leur part, Lereya, Winsper, Tang et Wolke (2017) ont démontré que la présence de cauchemars chroniques chez les jeunes adolescents était associée à la symptomatologie du TPL et que cette association était médiatisée par les difficultés émotionnelles et comportementales. Finalement, Stockdale, Coyne, Nelson et Erickson (2015) profitent de la popularité des médias sociaux chez 
les adolescents afin d'examiner les comportements en ligne des adolescents avec un TPL. Les chercheurs constatent que les caractéristiques du TPL étaient associées à des comportements de cyberintimidation, médiatisée par la présence de jalousie chez les adolescents. Cette étude ouvre ainsi la voie à l'exploration des comportements en ligne associés aux symptômes du TPL à l'adolescence, pouvant rendre plus facile l'identification des jeunes afin de leur donner les traitements nécessaires.

\section{Conclusion}

Cette recension systématique permet d'appuyer le concept du TPL à titre de diagnostic valide à l'adolescence. Ce groupe clinique semble bel et bien présent d'où la pertinence de ce diagnostic. Cependant, les critères du DSM-5 étant larges et englobants, il serait nécessaire de préciser certaines particularités symptomatiques, telles que les caractéristiques des idéations suicidaires et la présence d'une comorbidité complexe de troubles externalisés et internalisés.

Cette recension souligne la variété des symptômes étudiés chez les adolescents ayant un TPL. Elle permet également de constater les symptômes qui facilitent la différenciation des adolescents avec un TPL de ceux ayant un autre trouble psychiatrique, un autre trouble de personnalité ou les adolescents non psychiatrisés. La combinaison de troubles internalisés et externalisés revient fréquemment dans les études examinées, ainsi que quelques difficultés au niveau du concept de soi, de l'hypermentalisation et des symptômes psychotiques. Néanmoins, la littérature scientifique demeure restreinte au sujet des adolescents ayant le diagnostic de TPL. Il est nécessaire de continuer l'exploration de ce trouble chez cette population spécifique afin de pouvoir identifier plus rapidement et efficacement les jeunes ayant besoin de traitements ou de supports, ainsi que d'abolir les tabous à ce sujet. 
L'abus durant l'enfance étant l'une des causes le plus fréquemment mentionnée concernant le développement du TPL à l'âge adulte, plusieurs études mettent de l'avant l'idée que le TPL ne serait qu'une manifestation du syndrome de stress post-traumatique (SSPT). Plus précisément, certaines études associent le diagnostic du TPL au syndrome de stress post-traumatique complexe (CPTSD: complex post-traumatic stress disorder). Ce syndrome ajoute les critères de la perturbation de l'autorégulation émotionnelle (colère), d'un concept de soi négatif (culpabilité, sans valeur) et des difficultés relationnelles (déconnexion aux autres) et demeure un diagnostic différent du SSPT (Cloitre, Garvert, Weiss, Carlson et Bryant, 2014). Pourtant, l'abus durant l'enfance n'est pas une cause directe au développement du TPL. Le nombre de sujets ayant un TPL rapportant un traumatisme en bas âge est très variable d'une étude à l'autre et ne démontre pas un lien de causalité. Plusieurs personnes n'ayant pas vécu de traumatisme dans leur enfance développent néanmoins un TPL plus tard. Afin de démystifier cette controverse, l'étude des différences symptomatologiques et développementales chez les sujets TPL ayant vécu ou non un traumatisme durant l'enfance est nécessaire.

\section{Références}

American Psychiatric Association (1952). Diagnostic and Statistical Manual of Mental Disorders, First Edition: DSM-1. American Psychiatric Pub.

American Psychiatric Association (1968). Diagnostic and Statistical Manual of Mental Disorders, Second Edition: DSM-2. American Psychiatric Pub.

American Psychiatric Association (1980). Diagnostic and Statistical Manual of Mental Disorders, Third Edition: DSM-3. American Psychiatric Pub.

American Psychiatric Association (2000). Diagnostic and Statistical Manual of Mental Disorders, Fourth Edition: DSM-4-TR. American Psychiatric Pub. 
American Psychiatric Association. (2013). Diagnostic and statistical manual of mental disorders DSM-5 (5e éd.). Arlington, VA: American Psychiatric Pub.

Atlas, J.A. et Wolfson, M.A. (1996). Depression and dissociation as features of borderline personality disorder in hospitalized adolescents. Psychological Reports, 78, 624-626.

Bradley, R., Conklin, C.Z. et Westen, D. (2005). The borderline personality diagnosis in adolescents: gender differences and subtypes. Journal of Child Psychology and Psychiatry, 46(9), 1006-1019. Doi: 10.1111/j.1469-7610.2004.00401.x

Catthoor, K., Feenstar, D.J., Hutsebaut, J., Schrijvers, D. et Sabbe, B. (2015). Adolescents with personality disorders suffer from severe psychiatric stigma: Evidence from a sample of 131 patients. Adolescent Health, Medecine and Therapeutics, 6, 81-89.

Chabrol, H., Montovany, A., Callahan, S., Chouicha, K. et Ducongé, E. (2002). Factor analyses of the DIB-R in adolescents. Journal of Personality Disorders, 16(4), 374-384.

Chanen, A., Jovev, M. et Jackson, H.J. (2007). Adaptative functioning and psychiatric symptoms in adolescents with borderline personality disorder. Journal of Clinical Psychiatry, 68(2), 297306.

Chanen, A. et Kaess, M. (2012). Developmental pathways to borderline personality disorder. Current Psychiatry Reports, 14, 45-53. Doi: 10.1007/s11920-011-0242-y

Chanen, A. (2015). Borderline personality disorder in young people: Are we there yet? Journal of Clinical Psychology, 71, 778-791.

Cloitre, M., Garvert, D.W., Weiss, B., Carlson, E.B. et Bryant, R.A. (2014). Distinguishing PTSD, complex PTSD, and borderline personality disorder: A latent class analysis. European Journal of Psychotraumatology, 5. http://dx.doi.org/10.3402/ejpt.v5.25097 
Costa, P. T., Jr., et McCrae, R. R. (1994). Stability and change in personality from adolescence through adulthood. Dans: C. F. Halverson, Jr., G. A. Kohnstamm, et R. P. Martin (dir), The developing structure of temperament and personality from infancy to adulthood (pp. 139-150). Hillsdale, NJ: Lawrence Erlbaum Associates

Courtney-Seidler, E.A., Klein, D. et Miller, A.L. (2013). Borderline personality disorder in adolescents. Clinical Psychology: Science and Practice, 20(4), 425-444.

Deutsch, H. (1942). Some forms of emotional disturbance and their relationship to schizophrenia. The Psychoanalytic Quarterly, 11(3), 301-321.

https://doi.org/10.1080/21674086.1942.11925501

Fossati, A., Gratz, K.L., Maffei, C. et Barroni, S. (2013). Emotion dysregulation and impulsivity additively predict borderline personality disorder features in Italian nonclinical adolescents. Personality and Mental Health, 7, 320-333. Doi: 10.1002/pmh.1229

Goodman, M., Patil, U., Triebwasser, J., Diamond, E., Hiller, A., Hoffman, P., ..., New, A. (2010). Parental viewpoints of trajectories to borderline personality disorder in female offspring. Journal of Personality Disorders, 24(2), 204-216.

Goodman, M., Patel, U., Oakes, A., Matho, A. et Triebwasser, J. (2013). Developmental trajectories to male borderline personality disorder. Journal of Personality Disorders, 27(6), 764782.

Ha, C., Balderas, J.C., Zanarini, M.C., Oldham, J. et Sharp, C. (2014). Psychiatric comorbidity in hospitalized adolescents with borderline personality disorder. Journal of Clinical Psychiatry, 75(5), e457-e464. Doi: 10.4088/JCP.13m08696

Horesh, N., Orbach, I., Gothelf, D., Efratti, M. et Apter, A. (2003). Comparison of the suicidal behavior of adolescent inpatients with borderline personality disorder and major depression. Journal of Nervous and Mental Disease, 191(9), 582-588. Doi: 10/1097/01nmd.0000087184.56009.61 
Huynh, C., Guilé, J.M., Breton, J.J. et Godbout, R. (2016). Sleep-wake patterns of adolescents with borderline personality disorder and bipolar disorder. Child Psychiatry and Human Development, 47, 202-214. Doi: 10.1007/s10578-015-0557-8

Kaess, M., Brunner, R. et Chanen, A. (2014). Borderline personality disorder in adolescence. Pediatrics, 134, 782-793. doi: 10.1542/peds.2013-3677

Kernberg, O. (1966). Structural derivatives of object relationships. The International Journal of Psychoanalysis, 47, 236-253.

Kramer, U., Temes, C.M., Magni, L.R., Fitzmaurice, G.M., Aguirre, B.A., Goodman, M. et Zanarini, M.C. (2017). Psychosocial functioning in adolescents with and without borderline personality disorder. Personality and Mental Health, 11, 164-170. Doi: 10.1002/pmh.1377

Laporte, L. et Desrosiers, L. (2016). Le trouble de personnalité limite dans le milieu de la protection de la jeunesse : État des lieux. Source: http://observatoiremaltraitance.ca/Pages/Coupd\%27oeil-sur-le-trouble-de-la-personnalité-limite-dans-le-milieu-de-la-protection-de-lajeunesse.aspx $>$

Lawrence, K.A., Allen, J.S. et Chanen, A.M. (2011). A study of maladaptive schemas and borderline personality disorder in young people. Cognitive Therapy and Research, 35, 30-39. Doi: $10.1007 / \mathrm{s} 10608-009-9292-4$

Lereya, S.T., Winsper, C., Tang, N.K.Y. et Wolke, D. (2017). Sleep problems in childhood and borderline personality disorder symptoms in early adolescence. Journal of Abnormal Child Psychology, 45, 193-206. Doi: 10.1007/s10802-016-0158-4

Loas, G., Speranza, M., Pham-Scottez, A., Perez-Diaz, F. et Corcos, M. (2012). Alexithymia in adolescents with borderline personality disorder. Journal of Psychosomatic Research, 72, 147152. Doi: 10.1016/j.jpsychores.2011.11.006

Matusiewicz, A., Weaverling, G. et Lejuez, C.W. (2014). Emotion dysregulation among adolescents with borderline personality disorder. Dans: Sharp, C. et Tackett, J. (dir), Handbook 
of Borderline Personality Disorder in Children and Adolescents. NY, Springer, 177-194. https://doi.org/10.1007/978-1-4939-0591-1_13

McManus, M., Lerner, H., Robbins, D. et Barbour, C. (1984). Assessment of borderline symptomatology in hospitalized adolescents. Journal of the American Academy of Child Psychiatry, 23(6), 685-694.

Meijer, M. et Treffers, P.D.A. (1991). Borderline and schizotypal disorders in children and adolescents. The British Journal of Psychiatry, 158(2), 205-212.

https://doi.org/10.1192/bjp.158.2.205

Miller, A.L., Muehlenkamp, J.J. et Jacobson, C.M. (2008). Fact or fiction: Diagnosing borderline personality disorder in adolescents. Clinical Psychology Review, 28, 969-981. Doi:

10.1016/j.cpr.2008.02.004

New, A.S. et Triebwasser, J. (2017). A history of borderline personality disorder. Dans Stanley, B. et New, A. (dir), Borderline Personality Disorder (p.1-16). New-York, NY: Oxford University Press.

Oldham, J.M. (2006). Borderline personality disorder and suicidality. American Journal of Psychiatry, 163(1), 20-24.

Pinto, A., Grapentine, W.L., Francis, G. et Picariello, C.M. (1996). Borderline personality disorder in adolescents: Affective and cognitive features. Journal of the American Academy of Child and Adolescent Psychiatry, 35(10), 1338-1343.

Quek, J., Melvin, G.A., Bennett, C., Gordon, M.S., Saeedi, N. et Newman, L.K. (2018). Mentalization in adolescents with borderline personality disorder: A comparison with healthy controls. Journal of Personality Disorders, 32, 1-19.

Serman, N., Johnson, J.G., Geller, P.A., Kanost, R.E. et Zacharapoulou, H. (2002). Personality disoders associated with substance use among American and Greek adolescents. Adolescence, $37(148), 841-854$. 
Stern, A. (1938). Psychoanalytic investigation of and therapy in the border line group of neuroses. The Psychoanalytic Quarterly, 7(4), 467-489. Doi: 10.1080/21674086.1938.11925367

Stern, A. (1945). Psychoanalytic therapy in the borderline neuroses. The Psychoanalytic Quarterly, 14, 190-198.

Stockdale, L.A., Coyne, S.M., Nelson, D.A. et Erickson, D.H. (2015). Borderline personality disorder features, jealousy, and cyberbullying in adolescence. Personality and Individual Differences, 83, 148-153. https://dx.doi.org/10.1016/j.paid.2015.04.003

Venta, A., Ross, E., Schatte, D. et Sharp, C. (2012). Suicide ideation and attempts among inpatient adolescents with borderline personality disorder: Frequency, intensity and age of onset. Personality and Mental Health, 6, 340-351. Doi: 10.1002/pmh.1202

Winsper, C., Marwaha, S., Lereya, S.T., Thompson, A., Eyden, J. et Singh, S.P. (2015). Clinical and psychosocial outcomes of borderline personality disorder in childhood and adolescence: a systematic review. Psychological Medicine, 45, 2237-2251. Doi: 10.1017/S0033291715000318

World Health Organization. (1993). ICD-10: international statistical classification of diseases and related health problems. Geneva: World Health Organization

Yen, S., Gagnon, K. et Spirito, A. (2013). Borderline personality disorder in suicidal adolescents. Personality and Mental Health, 7, 89-101. Doi: 10.1002/pmh.1216

Zanarini, M.C., Temes, C.M., Magni, L.R., Fitzmaurice, G.M., Aguirre, B.A. et Goodman, M. (2017). Prevalence rates of borderline symptoms reported by adolescent inpatients with BPD, psychiatrically healthy adolescents and adult inpatients with BPD. Personality and Mental Health, 11, 150-156. Doi: 10.1002/pmh.1378 